ESAIM: M2AN 50 (2016) 263-288

DOI: $10.1051 / \mathrm{m} 2 \mathrm{an} / 2015042$
ESAIM: Mathematical Modelling and Numerical Analysis

www.esaim-m2an.org

\title{
DISCRETIZATION BY RATIONAL AND QUASI-RATIONAL FUNCTIONS OF MULTI-DIMENSIONAL ELLIPTIC PROBLEMS IN THE WHOLE SPACE
}

\author{
T.Z. Boulmezaoud ${ }^{1}$, N. Arar $^{2}$, N. Kerdid ${ }^{3}$ and A. Kourta ${ }^{2}$
}

\begin{abstract}
We propose a new spectral method for solving multi-dimensional second order elliptic equations with varying coefficients in the whole space. This method employs an orthogonal family of quasi-rational functions recently discovered by Arar and Boulmezaoud. After proving an error estimate, we present some computational tests which demonstrate the efficiency of the method and the significance of its developmental potential.
\end{abstract}

Mathematics Subject Classification. 65N35, 35A01, 35C10, 35C20, 65J99.

Received September 4, 2013. Revised March 4, 2015.

Published online January 28, 2016.

\section{INTRODUCTION}

Among the existing approaches for solving partial differential equations in unbounded regions, one can underline the emergence of spectral methods based on the use of polynomials (see, e.g., $[32,43]$ ), gaussian functions (see, e.g., $[33,39]$ ) or rational functions (see, e.g., $[17,18,22,37]$ ). Although it is frequently used in bounded domains, global approximation by polynomials has proven not efficient in unbounded regions, especially in the context of second order elliptic equations. This is chiefly due to the fact that solutions are often required to be bounded at infinity and do not have a polynomial growth. On the contrary, rational functions give the possibility to take into account the decay of functions at large distances, but their disadvantage is that they are often difficult to deploy. In any case, the description of the evanescence of quantities at infinity arises to be crucial in almost all practical problems in physics and engineering for which the considered region has an infinite extent.

Of course, other methods exist for discretizing PDEs in unbounded domains. The most popular approaches consist in truncating the computational domain in a reasonable way. This is typically the case for ABC (Absorbing Boundary Conditions) methods which date back to the sixties (see $[5,30,31,42]$ ). This is also the case for PML (Perfectly Matched Layer) methods (see [6,7]). There are also some methods which preserve the unboundedness of the domain. We can mention the so-called Boundary Element Methods (BEM) (see, e.g., [19, 26, 36]

Keywords and phrases. Unbounded domains, spectral methods, rational functions, approximation, the whole space.

1 Laboratoire de Mathématiques de Versailles, Université de Versailles Saint-Quentin-en-Yvelines 45, avenue des Etats-Unis, 78035, Versailles, cedex, France. tahar.boulmezaoud@uvsq.fr

2 Department of Mathematics, University Constantine 1, Constantine, Algeria. nour.arar@yahoo.fr; a.kourta@yahoo.fr

3 IMSIU, College of Sciences, Department of Mathematics and Statistics, PO-Box 90950, 11623 Riyadh, KSA.

nabil_kerdid@yahoo.fr 
and references therein), the BEF-FEM coupling methods (see, e.g., $[20,23,28,29,40]$ ), the IFEM (Inverted Finite Element Method) introduced by one of the authors of this paper (see [13] or [16]) and the IEM (Infinite Element Method) introduced first in [9] (see also [10,21] or [34] and references therein).

Our aim in this paper is to propose a multi-dimensional spectral method based on the use of a special kind of rational or quasirational functions for solving second order elliptic problems with varying coefficients in the whole space. The model problem is

$$
-\sum_{i, j=1}^{d} \frac{\partial}{\partial x_{i}} a_{i j}(x) \frac{\partial u}{\partial x_{j}}(x)+\sum_{i=1}^{d} b_{i}(x) \frac{\partial u}{\partial x_{i}}+c(x) u=f(x), x \in \mathbb{R}^{d},
$$

where $a_{i, j}, b_{i}, 1 \leq i, j \leq d$ and $c$ are variable coefficients and $f$ a given function of a certain regularity specified later. The approximation we propose is based on the use of functions of the form

$$
\sum_{m=0}^{N} \frac{p_{m}(x)}{\left(|x|^{2}+1\right)^{(2 m+d-2) / 2}}
$$

when $d \geq 2$, and of the form

$$
\sum_{m=0}^{N} \frac{p_{m}(x)}{\left(x^{2}+1\right)^{m / 2}}
$$

when $d=1$. In both the expressions $p_{m}, 0 \leq m \leq N$, is a polynomial function of degree less than or equal to $m$. In addition, when $d=1, p_{m}$ has the same parity as $m$, that is $p_{m}(-x)=(-1)^{m} p_{m}(x)$ for all $x \in \mathbb{R}$.

Furthermore, inspired by the work of Arar and Boulmezaoud [3], in which a special family of eigenfunctions of the operator $\left(1+|.|^{2}\right)^{-2} \Delta$ is discovered and given explicitly, we prove that the method is convergent and that error decreases algebraically for functions with a moderate decay at large distances. We also show that the use of orthogonal Arar-Boulmezaoud functions, defined by the forthcoming formula (3.13), makes the implementation of the method easier, especially for Poisson's equation for which the obtained linear system is diagonal.

The remaining is organized as follows. In Section 2, we recall some definitions and some properties of the underlying functional spaces. A weak formulation of equation (1.1) is given. Section 3 is devoted to the approximation of (1.1); we outline a new spectral method to approximate (1.1) and we prove its convergence. Besides this method, we propose the use of an adequate family of orthogonal rational or quasirational functions as a basis. Details of implementation and numerical results are given in Section 4.

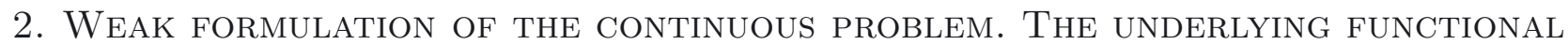 SPACES}

In what follows $d \geq 1$ denotes an integer. Given a real number $p, 1 \leq p<+\infty$, we denote as usual by $L^{p}\left(\mathbb{R}^{d}\right)$ (resp. $L^{\infty}\left(\mathbb{R}^{d}\right)$ ) the space of (equivalence classes of) all measurable functions that are $p$ th power integrable (resp. essentially bounded) on $\mathbb{R}^{d}$.

Given an arbitrary measurable and positive function $\rho, L_{\rho}^{\infty}\left(\mathbb{R}^{d}\right)$ denotes the space of measurable functions $v$ satisfying $\rho v \in L^{\infty}\left(\mathbb{R}^{d}\right)$. This space is equipped with the norm

$$
\|v\|_{L_{\rho}^{\infty}\left(\mathbb{R}^{d}\right)}=\|\rho v\|_{L^{\infty}\left(\mathbb{R}^{d}\right)} .
$$

For each integer $m \geq 0$ and each real $\alpha \geq 0$, define $\mathbb{W}_{\alpha}^{m}\left(\mathbb{R}^{d}\right)$ as the space of all the real measurable functions satisfying

$$
\left(|x|^{2}+1\right)^{(\alpha-m+|\lambda|) / 2} \partial^{\lambda} u \in L^{2}\left(\mathbb{R}^{d}\right),
$$


for any multi-index $\lambda \in \mathbb{N}^{d}$ such that $0 \leq|\lambda| \leq m$ (with $|\lambda|=\lambda_{1}+\ldots \lambda_{m}$ ). This is a Hilbert space equipped with the scalar product

$$
\langle u, v\rangle_{\mathbb{W}_{\alpha}^{m}\left(\mathbb{R}^{d}\right)}=\sum_{|\lambda| \leq m} \int_{\mathbb{R}^{d}}\left(|x|^{2}+1\right)^{\alpha-m+|\lambda|} \partial^{\lambda} u(x) . \partial^{\lambda} v(x) \mathrm{d} x .
$$

The reader interested in properties of the spaces $\mathbb{W}_{\alpha}^{m}$ can consult, e.g., $[2,11,12,15,35,38]$.

In the sequel, given $x=\left(x_{1}, \ldots, x_{d}\right) \in \mathbb{R}^{d}$, we set $\langle x\rangle=\left(|x|^{2}+1\right)^{1 / 2}=\left(x_{1}^{2}+\ldots x_{d}^{2}+1\right)^{1 / 2}$ and we consider the weight function

$$
w(x)= \begin{cases}\frac{1}{|x|^{2}+1} & \text { if } d \neq 2, \\ \frac{1}{\left(|x|^{2}+1\right)\left(\log \left(2+|x|^{2}\right)\right)^{2}} & \text { if } d=2 .\end{cases}
$$

Define $W_{w}^{1}\left(\mathbb{R}^{d}\right)$ as the space all the (generalized) functions $v$ satisfying

$$
\int_{\mathbb{R}^{d}} w(x)|v(x)|^{2} \mathrm{~d} x<\infty, \int_{\mathbb{R}^{d}}|\nabla v(x)|^{2} \mathrm{~d} x<\infty,
$$

and endowed with the norm

$$
\|v\|_{W_{w}^{1}\left(\mathbb{R}^{d}\right)}^{2}=\int_{\mathbb{R}^{d}} w(x)|v(x)|^{2} \mathrm{~d} x+\int_{\mathbb{R}^{d}}|\nabla v(x)|^{2} \mathrm{~d} x .
$$

Set $W_{w}^{-1}\left(\mathbb{R}^{d}\right)$ the dual of $W_{w}^{1}\left(\mathbb{R}^{d}\right)$. When $d \neq 2, W_{w}^{1}\left(\mathbb{R}^{d}\right)=\mathbb{W}_{0}^{1}\left(\mathbb{R}^{d}\right)$.

When $d \geq 3$, the semi-norm

$$
|v|_{W_{w}^{1}\left(\mathbb{R}^{d}\right)}=\left(\int_{\mathbb{R}^{d}}|\nabla v(x)|^{2} \mathrm{~d} x\right)^{1 / 2}
$$

is a norm on $W_{w}^{1}\left(\mathbb{R}^{d}\right)$ and there exists a constant $\kappa_{0}>0$ such that

$$
\forall v \in W_{w}^{1}\left(\mathbb{R}^{d}\right), \int_{\mathbb{R}^{d}} w(x)|v(x)|^{2} \mathrm{~d} x \leq \kappa_{0} \int_{\mathbb{R}^{d}}|\nabla v(x)|^{2} \mathrm{~d} x
$$

(see $[2,38]$ or $[3])$. When $d \in\{1,2\},(2.1)$ is no longer valid since constant functions belong to $W_{w}^{1}\left(\mathbb{R}^{d}\right)$. However, in the latter case there exists a positive constant still denoted by $\kappa_{0}$ such that

$$
\forall v \in W_{w}^{1}\left(\mathbb{R}^{d}\right), \inf _{k \in \mathbb{R}} \int_{\mathbb{R}^{d}} w(x)|v(x)-k|^{2} \mathrm{~d} x \leq \kappa_{0} \int_{\mathbb{R}^{d}}|\nabla v(x)|^{2} \mathrm{~d} x .
$$

The best value of $k$ in this inequality corresponds to the weighted mean value of $v$, given by

$$
k=\frac{1}{\bar{w}} \int_{\mathbb{R}^{d}} w(x) v(x) \mathrm{d} x, \text { with } \bar{w}=\int_{\mathbb{R}^{d}} w(x) \mathrm{d} x .
$$

Thus, one can rewrite (2.2) into the form

$$
\forall v \in W_{w}^{1}\left(\mathbb{R}^{d}\right), \int_{\mathbb{R}^{d}} w(x)|v(x)|^{2} \mathrm{~d} x \leq \kappa_{0} \int_{\mathbb{R}^{d}}|\nabla v(x)|^{2} \mathrm{~d} x+\frac{1}{\bar{w}}\left(\int_{\mathbb{R}^{d}} w(x) v(x) \mathrm{d} x\right)^{2} .
$$


Here and subsequently, we assume the following

$\left(\mathscr{H}_{1}\right) f$ belongs to $W_{w}^{-1}\left(\mathbb{R}^{d}\right)$. In particular, this assumption holds true when

$$
\int_{\mathbb{R}^{d}} \frac{|f(x)|^{2}}{w(x)} \mathrm{d} x<+\infty .
$$

$\left(\mathscr{H}_{2}\right)\left(a_{i j}\right)_{1 \leq i, j \leq d} \in L^{\infty}\left(\mathbb{R}^{d}\right)^{d \times d}$ and there exists a constant $\alpha>0$ such that

$$
\forall \xi=\left(\xi_{1}, \ldots, \xi_{d}\right) \in \mathbb{R}^{d}, \sum_{i, j=1}^{d} a_{i, j}(x) \xi_{i} \xi_{j} \geq \alpha|\xi|^{2} \text {, a.e. in } \mathbb{R}^{d} .
$$

$\left(\mathscr{H}_{3}\right) \boldsymbol{b}=\left(b_{1}, \ldots, b_{d}\right) \in L_{w^{-1 / 2}}^{\infty}\left(\mathbb{R}^{d}\right)^{d}, \operatorname{div} \boldsymbol{b} \in L_{w^{-1}}^{\infty}\left(\mathbb{R}^{d}\right)$ and $c \in L_{w^{-1}}^{\infty}\left(\mathbb{R}^{d}\right)$, that is there exists a constant $C>0$ such that

$$
|\boldsymbol{b}(x)|^{2}+|\operatorname{div} \boldsymbol{b}(x)|+|c(x)| \leq C w(x) \text { a.e. in } \mathbb{R}^{d} .
$$

$\left(\mathscr{H}_{4}\right)$ there exists a constant $\epsilon$ satisfying

$$
\epsilon>-\frac{\alpha}{\kappa_{0}} \text { if } d \geq 3, \epsilon>0 \text { if } d \in\{1,2\}
$$

and such that

$$
c(x)-\frac{1}{2} \operatorname{div} \boldsymbol{b}(x) \geq \epsilon w(x) \text { a.e. in } \mathbb{R}^{d} .
$$

Assumptions $\left(\mathscr{H}_{3}\right)$ and $\left(\mathscr{H}_{4}\right)$ are automatically fullfiled if $\boldsymbol{b}=\mathbf{0}, c=0$ and $d \geq 3$.

Let us now come back to equation (1.1). We look for solutions satisfying

$$
\int_{\mathbb{R}^{d}} w(x)|u(x)|^{2} \mathrm{~d} x<\infty, \int_{\mathbb{R}^{d}}|\nabla u(x)|^{2} \mathrm{~d} x<\infty .
$$

This problem can be rewritten into the weak form: find $u \in W_{w}^{1}\left(\mathbb{R}^{d}\right)$ such that

$$
\forall v \in W_{w}^{1}\left(\mathbb{R}^{d}\right), \mathscr{A}(u, v)=\langle f, v\rangle,
$$

with $\mathscr{A}$ the bilinear form

$$
\begin{aligned}
\mathscr{A}(u, v)= & \sum_{i, j=1}^{d} \int_{\mathbb{R}^{d}} a_{i, j}(x) \frac{\partial u}{\partial x_{i}}(x) \frac{\partial v}{\partial x_{j}}(x) \mathrm{d} x+\sum_{i=1}^{d} \int_{\mathbb{R}^{d}} b_{i}(x) \frac{\partial u}{\partial x_{j}}(x) v(x) \mathrm{d} x \\
& +\int_{\mathbb{R}^{d}} c(x) u(x) v(x) \mathrm{d} x .
\end{aligned}
$$

Assumptions $\left(\mathscr{H}_{2}\right)$ and $\left(\mathscr{H}_{3}\right)$ ensure the continuity of the bilinear form $\mathscr{A}(.,$.$) . Moreover, integration by parts$ gives

$$
\begin{aligned}
\mathscr{A}(v, v) & =\sum_{i, j=1}^{d} \int_{\mathbb{R}^{d}} a_{i, j}(x) \frac{\partial v}{\partial x_{i}}(x) \frac{\partial v}{\partial x_{j}}(x) \mathrm{d} x+\int_{\mathbb{R}^{d}}\left(c(x)-\frac{1}{2} \operatorname{div} b(x)\right) v(x)^{2} \mathrm{~d} x \\
& \geq \alpha \int_{\mathbb{R}^{d}}|\nabla v(x)|^{2} \mathrm{~d} x+\epsilon \int_{\mathbb{R}^{d}} w(x) v(x)^{2} \mathrm{~d} x,
\end{aligned}
$$

where we used assumptions $\left(\mathscr{H}_{2}\right),\left(\mathscr{H}_{3}\right)$ and $\left(\mathscr{H}_{4}\right)$ and Hardy inequality $(2.1)$. In view of Lax-Milgram theorem we get

Proposition 2.1. Assume that assumptions $\left(\mathscr{H}_{1}\right)-\left(\mathscr{H}_{4}\right)$ hold. Then, the problem (2.8) (or equivalently equation (1.1)) admits one and only one solution $u \in W_{w}^{1}\left(\mathbb{R}^{d}\right)$ and

$$
\|u\|_{W_{w}^{1}\left(\mathbb{R}^{d}\right)} \lesssim\|f\|_{W_{w}^{-1}\left(\mathbb{R}^{d}\right)} .
$$


The notation $a \lesssim b$ used in (2.9) means that there exists a constant $c$ independant of the involved functions such that $a \leq c b$.

When $d \leq 2$ and $b=c=0$, assumption $\left(\mathscr{H}_{4}\right)$ is not satisfied and Proposition 2.1 fails since uniqueness is lost (constant functions belong to $W_{w}^{1}\left(\mathbb{R}^{d}\right)$ ). In addition, existence is also lost except when $f$ satisfies the compatibility condition

$$
\langle f, 1\rangle=0 .
$$

This last condition is obtained by taking $v=1$ in $(2.8)$ (notice that $1 \in W_{w}^{1}\left(\mathbb{R}^{d}\right)$ when $d \leq 2$ ).

Thus, when $b=c=0$ and $d \in\{1,2\}$, equation (1.1) (or formulation (2.8)) may be completed with the following condition

$$
\int_{\mathbb{R}^{d}} w(x) u(x) \mathrm{d} x=0 .
$$

Then, problem (1.1) in combination with condition (2.11) can be written into the (equivalent) weak form: find $u \in W_{w}^{1}\left(\mathbb{R}^{d}\right)$ such that

$$
\forall v \in W_{w}^{1}\left(\mathbb{R}^{d}\right), \mathscr{A}_{\star}(u, v)=\langle f, v\rangle
$$

with

$$
\mathscr{A}_{\star}(u, v)=\mathscr{A}(u, v)+\kappa\left(\int_{\mathbb{R}^{d}} w(x) u(x) \mathrm{d} x\right)\left(\int_{\mathbb{R}^{d}} w(x) v(x) \mathrm{d} x\right),
$$

where henceforth $\kappa>0$ is a fixed constant.

Indeed, it suffices to observe that $\mathscr{A}(u, 1)=0$ for all $u \in W_{w}^{1}\left(\mathbb{R}^{d}\right)$.

The continuity of the bilinear form $\mathscr{A}_{\star}$, defined on $W_{w}^{1}\left(\mathbb{R}^{d}\right)^{2}$, is obvious. Its coercivity follows from inequality (2.3). By Lax-Milgram theorem, we get

Proposition 2.2. Suppose that $d \leq 2, b=c=0$ and that assumptions $\left(\mathscr{H}_{1}\right)$ and $\left(\mathscr{H}_{2}\right)$ hold. Then, problem (2.12) has a solution $u \in W_{w}^{1}\left(\mathbb{R}^{d}\right)$ if and only if $f$ satisfies (2.10). In that case, $u$ is unique and verifies

$$
\|u\|_{W_{w}^{1}\left(\mathbb{R}^{d}\right)} \lesssim\|f\|_{W_{w}^{-1}\left(\mathbb{R}^{d}\right)} .
$$

\section{Approximation by Rational And pSeUdo-Rational FUnCtions}

Our aim in this section is to outline general principles of the numerical method we propose and to examine its convergence. For the reader's convenience, we begin by introducing the finite dimensional spaces of quasi-rational functions we use in approximating the continuous problem.

\subsection{Discretization and error estimate}

We introduce a family of finite dimensional spaces $\left(H_{N}^{d}\right)_{\geq 0}$ as follows

- When $d=1$, the space $H_{N}^{d}$, is composed of functions of the form

$$
v(x)=\sum_{k=0}^{N} \frac{p_{k}(x)}{\left(x^{2}+1\right)^{k / 2}}, x \in \mathbb{R},
$$

where, for each $k \leq N, p_{k}$ is a polynomial function of degree less than or equal to $k$ and having the same parity as $k$, that is

$$
\forall x \in \mathbb{R}, p_{k}(-x)=(-1)^{k} p_{k}(x) .
$$

- When $d \geq 2$, the space $H_{N}^{d}$ is composed of functions of the form

$$
v(x)=\sum_{k=0}^{N} \frac{p_{k}(x)}{\left(|x|^{2}+1\right)^{(2 k+d-2) / 2}}, x \in \mathbb{R}^{d},
$$

where, for each $k \leq N, p_{k}$ is a polynomial of degree less than or equal to $k$. 
It is quite obvious that

$$
H_{0}^{d} \subset H_{1}^{d} \subset H_{2}^{d} \subset \ldots \subset H_{N}^{d} \subset \ldots
$$

Notice that $H_{N}^{d}$ is a subspace of $V_{N}^{d}$, with $H_{N}^{d} \neq V_{N}^{d}$, where $V_{N}^{d}$ is the space of functions $v$ of the form

$$
v(x)=\frac{P(x)}{\left(|x|^{2}+1\right)^{(2 k+d-2) / 2}}, x \in \mathbb{R}^{d},
$$

with $P$ a polynomial function of degree less than or equal to $2 N$. The functions of $H_{N}^{d}$ are obviously rational when the dimension $d$ is even. They are rational up to the multiplicative factor $\left(|x|^{2}+1\right)^{-1 / 2}$ when $d$ is odd. In the latter case, we say that functions of $H_{N}^{d}$ are quasi-rational.

The following inclusion holds for $d \geq 1$

$$
H_{N}^{d} \hookrightarrow W_{w}^{1}\left(\mathbb{R}^{d}\right) .
$$

When $d=1$, one can also prove that each $v \in H_{N}^{1}$ can be decomposed in a unique manner into the form

$$
v(x)=\sum_{k=0}^{[N / 2]} \frac{a_{k}}{\left(x^{2}+1\right)^{k}}+\sum_{k=0}^{[(N-1) / 2]} \frac{b_{k} x}{\left(x^{2}+1\right)^{k+1 / 2}},
$$

where $a_{k}$ and $b_{k}, 0 \leq k \leq N$, are real numbers with $b_{0}=0$ (one can write $x^{2}=\left(x^{2}+1\right)-1$ ). When $d \geq 2$, each $v \in H_{N}^{d}$ can be decomposed into the sum

$$
v(x)=\frac{a_{0}\left(x^{\prime}\right)}{\left(|x|^{2}+1\right)^{(d-2) / 2}}+\sum_{k=1}^{N} \frac{a_{k}\left(x^{\prime}\right)+b_{k}\left(x^{\prime}\right) x_{d}}{\left(|x|^{2}+1\right)^{(2 k+d-2) / 2}},
$$

where $x^{\prime}=\left(x_{1}, \ldots, x_{d-1}, 0\right)$ and for any $k \leq N, a_{k}$ and $b_{k}$ are two polynomial functions of degrees less than or equal to $k$ and to $k-1$ respectively. In view of these decompositions, we get

Lemma 3.1 (See also [3]). For all $k \geq 1$,

$$
\operatorname{dim} H_{N}^{d}=\left\{\begin{array}{c}
N+1 \\
\left(\begin{array}{c}
d+N \\
d
\end{array}\right)+\left(\begin{array}{c}
d+N-1 \\
d
\end{array}\right) \text { if } d=1,
\end{array}\right.
$$

Thus, when $d \geq 2$

$$
\operatorname{dim} H_{N}^{d} \sim \frac{2}{d !} N^{d}
$$

We now focus attention on the variational problem $(2.8)$ when assumptions $\left(\mathscr{H}_{1}\right)-\left(\mathscr{H}_{4}\right)$ are satisfied.

The discretized problem writes: find $u_{N} \in H_{N}^{d}$ such that

$$
\forall v_{N} \in H_{N}^{d} \quad \mathscr{A}\left(u_{N}, v_{N}\right)=\left\langle f, v_{N}\right\rangle,
$$

where $N \geq 1$ denotes an integer designated to tend to infinity. This is a Galerkin problem which can be reduced to a linear system of the form $A U_{N}=B_{N}$. And, as $H_{N}^{d} \hookrightarrow W_{w}^{1}\left(\mathbb{R}^{d}\right)$ and $\mathscr{A}$ is coercive on $W_{w}^{1}\left(\mathbb{R}^{d}\right)$, the matrix $A$ is invertible and the discrete problem (3.7) clearly admits a unique solution $u_{N} \in H_{N}^{d}$.

Theorem 3.2. Suppose that assumptions $\left(\mathscr{H}_{1}\right)-\left(\mathscr{H}_{4}\right)$ are fulfilled. Let $u \in W_{w}^{1}\left(\mathbb{R}^{d}\right)\left(\right.$ resp. $\left.u_{N} \in H_{N}^{d}\right)$ be the unique solution of (2.8) (resp. of (3.7)). Assume that $u \in \mathbb{W}_{2 k}^{k+1}\left(\mathbb{R}^{d}\right)$ for some $k \geq 1$. Then, the following error estimate holds

$$
\left\|u-u_{N}\right\|_{W_{w}^{1}\left(\mathbb{R}^{d}\right)} \lesssim N^{-k}\|u\|_{\mathbb{W}_{2 k}^{k+1}\left(\mathbb{R}^{d}\right)},
$$


When $b=c=0$ and $d \leq 2$, problem (1.1) can be completed with condition (2.11) to ensure uniqueness. In the latter case, the well-posedness of the weak formulation (2.12) is guaranteed by Proposition 2.2, under assumptions $\left(\mathscr{H}_{1}\right)$ and $\left(\mathscr{H}_{2}\right)$. The corresponding discretized problem writes: find $u_{N} \in H_{N}^{d}$ such that

$$
\forall v_{N} \in H_{N}^{d} ; \mathscr{A}_{\star}\left(u_{N}, v_{N}\right)=\left\langle f, v_{N}\right\rangle .
$$

Theorem 3.3. Suppose that $d \leq 2, b=c=0$ and that assumptions $\left(\mathscr{H}_{1}\right),\left(\mathscr{H}_{2}\right)$ and $(2.10)$ are fulfilled. Let $u \in W_{w}^{1}\left(\mathbb{R}^{d}\right)$ (resp. $\left.u_{N} \in H_{N}^{d}\right)$ be the unique solution of $(2.12)$ (resp. of (3.9)) and assume that $u \in \mathbb{W}_{2 k}^{k+1}\left(\mathbb{R}^{d}\right)$ for some $k \geq 1$. Then, conclusion of Theorem 3.2 remains true.

It is worth noting that, in the latter case, the discrete solution $u_{N}$ satisfies

$$
\int_{\mathbb{R}^{d}} w(x) u_{N}(x) \mathrm{d} x=0 .
$$

(Indeed, it suffices to choose $v=1$ in the weak formulation (3.9)).

The proofs of Theorems 3.2 and 3.3 are postponed to Section 3.3.

Error estimate (3.8) shows that the convergence depends not only on the smoothness of the function $u$ but also on its decay when $|x| \rightarrow+\infty$; it says that if $u$ is in $H_{\mathrm{loc}}^{k+1}$ and if its derivatives decrease sufficiently so that the integrals

$$
\forall|\mu| \leq k, \int_{\mathbb{R}^{d}}\left(|x|^{2}+1\right)^{k+|\mu|}\left|\partial^{\mu} u(x)\right|^{2} \mathrm{~d} x
$$

are finite, then the method is algebraically convergent and the error in $W_{w}^{1}$ norm decreases at least as $N^{-k}$. As a result, if $u$ is analytic and has an exponential decay at large distances, then the convergence has an infinite order since the error decreases faster than $1 / N^{k}$ for any finite order $k \geq 1$. One can surmise that for such functions the convergence is exponential. Of course, the method is exact (the error vanishes) if $u$ is quasi-rational of the form (3.1) when $d=1$ or (3.2) when $d \geq 2$ and if $N$ is sufficiently large.

\subsection{An orthogonal basis: Arar-Boulmezaoud functions}

Before proving estimate (3.8), we introduce an appropriate basis of $H_{N}^{d}$. The choice of this basis will play a prominent role not only in the proof, but also in computing the linear system arising from problem (3.7) (or problem (3.9)). Certainly, in view of decomposition (3.4), it may be tempting, when $d \geq 2$, to consider the functions

$$
\begin{gathered}
q_{2 k}(x)=\frac{\ell_{k}(x)}{\left(|x|^{2}+1\right)^{(2 k+d-2) / 2}}, 0 \leq k \leq N, \\
q_{2 k+1}(x)=\frac{\ell_{k-1}(x) x_{d}}{\left(|x|^{2}+1\right)^{(2 k+d-2) / 2}}, 1 \leq k \leq N,
\end{gathered}
$$

where $\left(\ell_{k}\right)_{0 \leq k \leq N}$ are $N+1$ polynomials satisfying the property $\operatorname{deg}\left(\ell_{k}\right)=k$ for $k \leq N$. When $d=1$, one can consider the functions

$$
q_{k}(x)=\frac{x^{k-2[k / 2]}}{\left(x^{2}+1\right)^{k / 2}}, 0 \leq k \leq N .
$$

For multiple practical reasons, it is more appropriate to use a special family of functions discovered by Arar and Boulmezaoud in [3]. These functions turn out to be suitable in proving error estimates and in the numerical resolution of the discrete problem (3.7) (or (3.9)). This is mainly due to their completeness and to their orthogonality properties.

In the sequel, we define the integers $\alpha_{\ell}, \ell \geq 0$, as follows:

- If $d=1$, then $\alpha_{\ell}=1$ for all $\ell \geq 0$, 
- if $d \geq 2$, then $\alpha_{\ell}$ is the dimension of the space $\mathbb{H}_{\ell}^{d}$ of spherical harmonics of degree $\ell$ on the unit sphere $\mathbb{S}^{d}:=\left\{x \in \mathbb{R}^{d+1}|| x \mid=1\right\}$ (spherical harmonics of degree $\ell$ on $\mathbb{S}^{d}$ are restrictions to $\mathbb{S}^{d}$ of harmonic homogeneous polynomials of degree $\ell$ on $\mathbb{R}^{d+1}$. See, e.g., [48] or [45]). We know that $\alpha_{0}=1, \alpha_{1}=d+1$ and for $\ell \geq 2$

$$
\alpha_{\ell}=\left(\begin{array}{c}
d+\ell \\
d
\end{array}\right)-\left(\begin{array}{c}
d+\ell-2 \\
d
\end{array}\right)=\left(\begin{array}{c}
d+\ell-1 \\
\ell
\end{array}\right)+\left(\begin{array}{c}
d+\ell-2 \\
\ell-1
\end{array}\right)
$$

The following identity will play an important role

$$
\operatorname{dim} H_{N}^{d}=\sum_{\ell=0}^{N} \alpha_{\ell}
$$

For each integer $\ell \geq 0$, we denote by $\left(\mathscr{Y}_{\ell, m}\right)_{\ell \geq 0,1 \leq m \leq \alpha_{\ell}}$ a basis of $\mathbb{H}_{\ell}^{d}$ chosen orthogonal with respect to the $L^{2}\left(\mathbb{S}^{d}\right)$ scalar product. That is, for all integers $\ell, \ell^{\prime} \geq 0, m, m^{\prime} \geq 1$, with $m \leq \alpha_{\ell}, m^{\prime} \leq \alpha_{\ell^{\prime}}$ and $(\ell, m) \neq\left(\ell^{\prime}, m^{\prime}\right)$, we have

$$
\int_{\mathbb{S}^{d}} \mathscr{Y}_{\ell, m} \cdot \mathscr{Y}_{\ell^{\prime}, m^{\prime}} \mathrm{d} S=0
$$

The Arar-Boulmezaoud functions are defined as follows: for each $\ell \geq 0$ and $1 \leq m \leq \alpha_{\ell}$

$$
\mathscr{W}_{\ell, m}(x)= \begin{cases}\left(\frac{2}{|x|^{2}+1}\right)^{\frac{d-2}{2}} \mathscr{Y}_{\ell, m}\left(\pi^{-1}(x)\right) & \text { if } d \geq 2, \\ (-1)^{\ell} B_{\ell}\left(x^{2}+1\right)^{1 / 2} \cos ((\ell+1) \arctan (x)+\ell \pi / 2) & \text { if } d=1 .\end{cases}
$$

where $1 \leq m \leq \alpha_{\ell}$ for $\ell \geq 0$. The normalization constants $B_{\ell}$ are given by

$$
B_{0}=1 \text { and } B_{\ell}=\frac{\sqrt{2}}{\sqrt{\ell(\ell+2) \pi}} \text { for } \ell \geq 1
$$

Here $\pi$ denotes the stereographic projection defined from $\mathbb{S}_{\star}^{d}=\mathbb{S}^{d}-\left\{(0, \ldots, 0,1)\right.$ onto $\mathbb{R}^{d}$ by

$$
\begin{aligned}
\pi: \mathbb{S}_{\star}^{d} & \longrightarrow \mathbb{R}^{d} \\
\xi & \longmapsto\left(\frac{\xi_{1}}{1-\xi_{d+1}}, \frac{\xi_{2}}{1-\xi_{d+1}}, \ldots, \frac{\xi_{d}}{1-\xi_{d+1}}\right) .
\end{aligned}
$$

Its inverse is given by

$$
\begin{aligned}
\pi^{-1}: \mathbb{R}^{d} & \longrightarrow \mathbb{S}_{\star}^{d} \\
x & \longmapsto\left(\frac{2 x_{1}}{|x|^{2}+1} \ldots, \frac{2 x_{d}}{|x|^{2}+1}, \frac{|x|^{2}-1}{|x|^{2}+1}\right) .
\end{aligned}
$$

In the one dimensional case $(d=1)$, we can also write

$$
(-1)^{\ell} \mathscr{W}_{\ell, 1}(x)= \begin{cases}B_{\ell} \sqrt{1+x^{2}} T_{\ell+1}\left(\frac{1}{\sqrt{x^{2}+1}}\right) & \text { if } \ell \text { is even, } \\ B_{\ell} x U_{\ell}\left(\frac{1}{\sqrt{x^{2}+1}}\right) & \text { if } \ell \text { is odd }\end{cases}
$$

where the constants $\left(B_{\ell}\right)_{\ell \geq 0}$ are given by (3.14), while $T_{\ell}$ and $U_{\ell}$ are Chebyshev polynomials of the first and second kind satisfying

$$
\forall \theta \in \mathbb{R}, \cos (\ell \theta)=T_{\ell}(\cos \theta), \sin ((\ell+1) \theta)=U_{\ell}(\cos \theta) \sin \theta
$$


We can also observe that

$$
\mathscr{W}_{\ell, 1}(x)=B_{\ell} \sqrt{x^{2}+1} \sin [(\ell+1)(\arctan (x)+\pi / 2)] .
$$

Thus,

$$
\mathscr{W}_{\ell, 1}(x)=(-1)^{\ell} B_{\ell} U_{\ell}\left(\frac{x}{\sqrt{x^{2}+1}}\right)
$$

When $d=2, \alpha_{\ell}=2 \ell+1$ for $\ell \geq 0$, and we can write

$$
\mathscr{W}_{\ell, m}(x)=Y_{\ell, m}\left(\pi^{-1}(x)\right), 0 \leq m \leq \ell, \mathscr{W}_{\ell, \ell+m}(x)=Z_{\ell, m}\left(\pi^{-1}(x)\right), 1 \leq m \leq \ell,
$$

where $Y_{\ell, m}$ and $Z_{\ell, m}$ are usual real spherical harmonics on $\mathbb{S}^{2}$ (see Appendix A). Lastly, in the three-dimensional case $(d=3)$ we can choose the following re-indexed family

$$
\begin{gathered}
\mathscr{W}_{\ell, k, j}(x)=\left(\frac{2}{|x|^{2}+1}\right)^{\frac{1}{2}} U_{\ell k j}\left(\pi^{-1}(x)\right), 0 \leq j \leq k \leq \ell, \\
\mathscr{W}_{\ell, k, k+j}(x)=\left(\frac{2}{|x|^{2}+1}\right)^{\frac{1}{2}} S_{\ell k j}\left(\pi^{-1}(x)\right), 1 \leq j \leq k \leq \ell,
\end{gathered}
$$

$\left(\alpha_{\ell}=(\ell+1)^{2}\right)$, with $U_{\ell k j}$ and $S_{\ell k j}$ real spherical harmonics on $\mathbb{S}^{3}$ (see Appendix A). It is worth noting that in the three cases $(d=1,2,3)$, the family constructed above is orthonormal.

Let us now recall some additional properties (see [3])

- For $\ell \geq 0$ and $1 \leq m \leq \alpha_{\ell}, \mathscr{W}_{\ell, m} \in H_{N}^{d}$ and

$$
-\Delta \mathscr{W}_{\ell, m}=\lambda_{\ell}\left(|x|^{2}+1\right)^{-2} \mathscr{W}_{\ell, m}
$$

where

$$
\lambda_{\ell}= \begin{cases}4 \ell(\ell+d-1)+d(d-2) & \text { if } d \geq 2 \\ \ell(\ell+2) & \text { if } d=1\end{cases}
$$

- $\left(\mathscr{W}_{\ell, m}\right)_{\ell \geq 0,1 \leq m \leq \alpha_{\ell}}$ is an orthogonal basis of $\mathbb{W}_{-2}^{0}\left(\mathbb{R}^{d}\right)$ satisfying: if $(\ell, m) \neq(j, s)$

$$
\int_{\mathbb{R}^{d}} \frac{\mathscr{W}_{\ell, m}(x) \mathscr{W}_{j, s}(x)}{\left(|x|^{2}+1\right)^{2}} \mathrm{~d} x=0, \int_{\mathbb{R}^{d}} \nabla \mathscr{W}_{\ell, m}(x) \cdot \nabla \mathscr{W}_{j, s}(x) \mathrm{d} x=0 .
$$

The following lemma is crucial and follows from formula (3.11) (see also [3])

Lemma 3.4. $\left(\mathscr{W}_{\ell, m}\right)_{0 \leq \ell \leq N, 1 \leq m \leq \alpha_{\ell}}$ is a basis of $H_{N}^{d}$.

A direct consequence of this lemma and formula (3.17) is that the spaces $H_{N}^{d}, N \geq 0$, are stable under the action of the weighted Laplacian $\left(|\cdot|^{2}+1\right)^{2} \Delta$, that is

$$
\forall v_{N} \in H_{N}^{d},\left(|\cdot|^{2}+1\right)^{2} \Delta v_{N} \in H_{N}^{d} .
$$

\subsection{Proof of Theorems 3.2 and 3.3}

We now focus attention on the Proof of Theorem 3.2. The Proof of Theorem 3.3 is quite similar.

We denote by $\Pi_{N}$ the orthogonal projection from $\mathbb{W}_{-2}^{0}\left(\mathbb{R}^{d}\right)$ on $H_{N}^{d}$. Recall that $\mathbb{W}_{-2}^{0}\left(\mathbb{R}^{d}\right)$ is equipped with the inner product

$$
\langle v, w\rangle_{\mathbb{W}_{-2}^{0}\left(\mathbb{R}^{d}\right)}=\int_{\mathbb{R}^{d}} \frac{v(x) w(x)}{\left(|x|^{2}+1\right)^{2}} \mathrm{~d} x .
$$

In other words, for $w \in \mathbb{W}_{-2}^{0}\left(\mathbb{R}^{d}\right), \Pi_{N} w$ belongs to $H_{N}^{d}$ and satisfies

$$
\forall \varphi \in H_{N}^{d}, \int_{\mathbb{R}^{d}} \frac{\left(w-\Pi_{N} w\right)(x) \varphi(x)}{\left(|x|^{2}+1\right)^{2}} \mathrm{~d} x=0 .
$$


We have

Lemma 3.5. Let $u \in \mathbb{W}_{2 k}^{k+1}\left(\mathbb{R}^{d}\right)$ for some $k \geq 1$. Then,

$$
\begin{aligned}
\left\|\langle x\rangle^{-2}\left(u-\Pi_{N} u\right)\right\|_{L^{2}\left(\mathbb{R}^{d}\right)}^{2} & \lesssim N^{-k-1}\|u\|_{\mathbb{W}_{2 k}^{k+1}\left(\mathbb{R}^{d}\right)}, \\
\left\|\nabla\left(u-\Pi_{N} u\right)\right\|_{L^{2}\left(\mathbb{R}^{d}\right)} & \lesssim N^{-k}\|u\|_{\mathbb{W}_{2 k}^{k+1}\left(\mathbb{R}^{d}\right)} .
\end{aligned}
$$

Proof. Let $\left(u_{\ell, m}\right)_{\ell \geq 0,1 \leq m \leq \alpha_{\ell}}$ be the decomposition coefficients of $u$ on the basis $\left(\mathscr{W}_{\ell, m}\right)$, that is

$$
u=\sum_{\ell=0}^{+\infty} \sum_{m=1}^{\alpha_{\ell}} u_{\ell, m} \mathscr{W}_{\ell, m}
$$

It follows that

$$
\Pi_{N} u=\sum_{\ell=0}^{N} \sum_{m=1}^{\alpha_{\ell}} u_{\ell, m} \mathscr{W}_{\ell, m} .
$$

Consider the operator $\mathscr{L}$ defined formally as

$$
\forall v \in \mathscr{D}^{\prime}\left(\mathbb{R}^{d}\right), \mathscr{L} v=-\left(1+|\cdot|^{2}\right)^{2} \Delta v .
$$

We know on the one hand that the Laplace operator is continuous from $\mathbb{W}_{m}^{k+2}\left(\mathbb{R}^{d}\right)$ into $\mathbb{W}_{m}^{k}\left(\mathbb{R}^{d}\right)$ for all $k \geq 0$ and $m \in \mathbb{Z}$ (see, e.g., [2]). We also know that the operator $w \mapsto\left(1+|.|^{2}\right)^{2} w$ is continuous from $\mathbb{W}_{m}^{k}\left(\mathbb{R}^{d}\right)$ into $\mathbb{W}_{m-4}^{k}\left(\mathbb{R}^{d}\right)$. It follows that $\mathscr{L}$ is linear continuous from $\mathbb{W}_{m}^{k+2}\left(\mathbb{R}^{d}\right)$ into $\mathbb{W}_{m-4}^{k}\left(\mathbb{R}^{d}\right)$ for $k \geq 0$ and $m \in \mathbb{Z}$. Moreover,

$$
\forall w, v \in \mathbb{W}_{2}^{2}\left(\mathbb{R}^{d}\right),\langle\mathscr{L} v, w\rangle_{\mathbb{W}_{-2}^{0}\left(\mathbb{R}^{d}\right)}=\langle v, \mathscr{L} w\rangle_{\mathbb{W}_{-2}^{0}\left(\mathbb{R}^{d}\right)} .
$$

In view of (3.17) and (3.18) we have

$$
\begin{aligned}
\left\|u-\Pi_{N} u\right\|_{\mathbb{W}_{-2}^{0}\left(\mathbb{R}^{d}\right)}^{2} & =\left\|\langle x\rangle^{-2}\left(u-\Pi_{N} u\right)\right\|_{L^{2}\left(\mathbb{R}^{d}\right)}^{2} \\
& =\sum_{\ell=N+1}^{+\infty} \sum_{m=1}^{\alpha_{\ell}}\left|u_{\ell, m}\right|^{2}\left\|\langle x\rangle^{-2} \mathscr{W}_{\ell, m}\right\|_{L^{2}\left(\mathbb{R}^{d}\right)}^{2} \\
\left\|\nabla\left(u-\Pi_{N} u\right)\right\|_{L^{2}\left(\mathbb{R}^{d}\right)}^{2} & =\sum_{\ell=N+1}^{+\infty} \sum_{m=1}^{\alpha_{\ell}}\left|u_{\ell, m}\right|^{2}\left\|\nabla \mathscr{W}_{\ell, m}\right\|_{L^{2}\left(\mathbb{R}^{d}\right)}^{2}, \\
& =\sum_{\ell=N+1}^{+\infty} \sum_{m=1}^{\alpha_{\ell}} \lambda_{\ell}\left|u_{\ell, m}\right|^{2}\left\|\langle x\rangle^{-2} \mathscr{W}_{\ell, m}\right\|_{L^{2}\left(\mathbb{R}^{d}\right)}^{2} .
\end{aligned}
$$

At this stage, two cases are distinguished

Case 1. $k$ is odd. Suppose that $k=2 s+1$ for some $s \geq 0$ and set $v=\mathscr{L}^{s+1} u$. Since $u \in \mathbb{W}_{2 k}^{k+1}\left(\mathbb{R}^{d}\right)=\mathbb{W}_{4 s+2}^{2 s+2}\left(\mathbb{R}^{d}\right)$, we deduce that $\mathscr{L} u \in \mathbb{W}_{4(s-1)+2}^{2 s}\left(\mathbb{R}^{d}\right), \ldots, v=\mathscr{L}^{s+1} u \in \mathbb{W}_{-2}^{0}\left(\mathbb{R}^{d}\right)$ and

$$
v=\sum_{\ell=0}^{+\infty} \sum_{m=1}^{\alpha_{\ell}} \lambda_{\ell}^{s+1} u_{\ell, m} \mathscr{W}_{\ell, m} .
$$

In fact, using formulae (3.21) and (3.17) gives

$$
\left\langle\mathscr{L}^{s+1} u, \mathscr{W}_{\ell, m}\right\rangle_{\mathbb{W}_{-2}^{0}\left(\mathbb{R}^{d}\right)}=\left\langle u, \mathscr{L}^{s+1} \mathscr{W}_{\ell, m}\right\rangle_{\mathbb{W}_{-2}^{0}\left(\mathbb{R}^{d}\right)}=\lambda_{\ell}^{s+1}\left\langle u, \mathscr{W}_{\ell, m}\right\rangle_{\mathbb{W}_{-2}^{0}\left(\mathbb{R}^{d}\right)} .
$$

It follows that

$$
\Pi_{N} v=\sum_{\ell=0}^{N} \sum_{m=1}^{\alpha_{\ell}} \lambda_{\ell}^{s+1} u_{\ell, m} \mathscr{W}_{\ell, m} .
$$


In view of (3.18) we have

$$
\begin{aligned}
\left\|v-\Pi_{N} v\right\|_{\mathbb{W}_{-2}^{0}\left(\mathbb{R}^{d}\right)}^{2} & =\sum_{\ell=N+1}^{+\infty} \sum_{m=1}^{\alpha_{\ell}} \lambda_{\ell}^{2 s+2}\left|u_{\ell, m}\right|^{2}\left\|\langle x\rangle^{-2} \mathscr{W}_{\ell, m}\right\|_{L^{2}\left(\mathbb{R}^{d}\right)}^{2} \\
& \geq \lambda_{N+1}^{2 s+2}\left\|\langle x\rangle^{-2}\left(u-\Pi_{N} u\right)\right\|_{L^{2}\left(\mathbb{R}^{d}\right)}^{2} \\
& \geq C_{1} N^{2 k+2}\left\|u-\Pi_{N} u\right\|_{\mathbb{W}_{-2}^{0}\left(\mathbb{R}^{d}\right)}^{2}
\end{aligned}
$$

We also have

$$
\begin{aligned}
\left\|v-\Pi_{N} v\right\|_{W_{-2}^{0}\left(\mathbb{R}^{d}\right)}^{2} & \geq \lambda_{N+1}^{2 s+1}\left\|\nabla\left(u-\Pi_{N} u\right)\right\|_{L^{2}\left(\mathbb{R}^{d}\right)}^{2} \\
& \geq C_{2} N^{2 k}\left\|\nabla\left(u-\Pi_{N} u\right)\right\|_{L^{2}\left(\mathbb{R}^{d}\right)}^{2}
\end{aligned}
$$

On the other hand,

$$
\left\|v-\Pi_{N} v\right\|_{W_{-2}^{0}\left(\mathbb{R}^{d}\right)}^{2} \leq\|v\|_{W_{-2}^{0}\left(\mathbb{R}^{d}\right)} \lesssim\|u\|_{W_{2 k}^{k+1}\left(\mathbb{R}^{d}\right)} .
$$

This ends the Proof of Lemma 3.5 when $k=2 s+1$.

Case 2. $k$ is even. Suppose that $k=2 s$ for some $s \geq 1$ and set $v=\mathscr{L}^{s} u$. Then, $v \in \mathbb{W}_{0}^{1}\left(\mathbb{R}^{d}\right)$. And, as before, we have

$$
\begin{aligned}
\|\nabla v\|_{L^{2}\left(\mathbb{R}^{d}\right)}^{2} & =\sum_{\ell=0}^{+\infty} \sum_{m=1}^{\alpha_{\ell}} \lambda_{\ell}^{2 s}\left|u_{\ell, m}\right|^{2}\left\|\nabla \mathscr{W}_{\ell, m}\right\|_{L^{2}\left(\mathbb{R}^{d}\right)}^{2} \\
& =\sum_{\ell=0}^{+\infty} \sum_{m=1}^{\alpha_{\ell}} \lambda_{\ell}^{2 s+1}\left|u_{\ell, m}\right|^{2}\left\|\langle x\rangle^{-2} \mathscr{W}_{\ell, m}\right\|_{L^{2}\left(\mathbb{R}^{d}\right)}^{2} \\
& \geq \lambda_{N+1}^{2 s+1}\left\|\langle x\rangle^{-2}\left(u-\Pi_{N} u\right)\right\|_{L^{2}\left(\mathbb{R}^{d}\right)}^{2} \\
& \geq C_{1}^{\prime} N^{2 k+2}\left\|\langle x\rangle^{-2}\left(u-\Pi_{N} u\right)\right\|_{L^{2}\left(\mathbb{R}^{d}\right)}^{2}
\end{aligned}
$$

Likewise,

$$
\begin{aligned}
\|\nabla v\|_{L^{2}\left(\mathbb{R}^{d}\right)}^{2} & \geq \lambda_{N+1}^{2 s}\left\|\nabla\left(u-\Pi_{N} u\right)\right\|_{L^{2}\left(\mathbb{R}^{d}\right)}^{2 s} \\
& \geq C_{2}^{\prime} N^{2 k}\left\|\nabla\left(u-\Pi_{N} u\right)\right\|_{L^{2}\left(\mathbb{R}^{d}\right)}^{2} .
\end{aligned}
$$

On the other hand,

$$
\|\nabla v\|_{L^{2}\left(\mathbb{R}^{d}\right)} \leq\|v\|_{\mathbb{W}_{0}^{1}\left(\mathbb{R}^{d}\right)} \lesssim\|u\|_{W_{2 k}^{k+1}\left(\mathbb{R}^{d}\right)} .
$$

This ends the proof of Lemma 3.5 when $k$ is even.

In order to finalize the proof of Theorem 3.2, we use Céa lemma (see [24] or, e.g., [25] or [8])

$$
\left\|u-u_{N}\right\|_{W_{w}^{1}\left(\mathbb{R}^{d}\right)} \lesssim \inf _{w \in H_{N}^{d}}\|u-w\|_{W_{w}^{1}\left(\mathbb{R}^{d}\right)} .
$$

Let

$$
u_{N}^{\star}= \begin{cases}\Pi_{N} u & \text { if } d \geq 3 \\ \Pi_{N} u-c_{N} & \text { if } d \leq 2\end{cases}
$$

where $c_{N}$ is a constant chosen such that

$$
\int_{\mathbb{R}^{d}} w(x)\left(u-u_{N}^{\star}\right) \mathrm{d} x=0 .
$$

Combining inequality (3.22) with (2.1) or with (2.3) we find

$$
\begin{aligned}
\left\|u-u_{N}\right\|_{W_{w}^{1}\left(\mathbb{R}^{d}\right)} & \lesssim\left\|u-u_{N}^{\star}\right\|_{W_{w}^{1}\left(\mathbb{R}^{d}\right)} \\
& \lesssim\left\|\nabla\left(u-u_{N}^{\star}\right)\right\|_{L^{2}\left(\mathbb{R}^{d}\right)}=\left\|\nabla\left(u-\Pi_{N} u\right)\right\|_{L^{2}\left(\mathbb{R}^{d}\right)} \\
& \lesssim N^{-k}\|u\|_{\mathbb{W}_{2 k}^{k+1}\left(\mathbb{R}^{d}\right)} .
\end{aligned}
$$

This ends the Proof of Theorem 3.2. 


\subsection{The case of Poisson's equation}

A remarkable property of the method concerns Poisson's equation

$$
-\Delta u=f \text { in } \mathbb{R}^{d}
$$

which corresponds to the case $a_{i, j}=\delta_{i, j}, b_{i}=0$ for $1 \leq i, j \leq d$ and $c=0$. A direct consequence of orthogonality properties of functions $\left(\mathscr{W}_{\ell, m}\right)$ is that the discretized system (3.7) as well as the continuous one (3.23) can be diagonalized. We set

$$
\eta_{\ell, m}=\int_{\mathbb{R}^{d}}\left|\nabla \mathscr{W}_{\ell, m}(x)\right|^{2} \mathrm{~d} x=\lambda_{\ell} \int_{\mathbb{R}^{d}}\left(|x|^{2}+1\right)^{-2}\left|\mathscr{W}_{\ell, m}(x)\right|^{2} \mathrm{~d} x,
$$

for $\ell \geq 0$ and $1 \leq m \leq \alpha_{\ell}$. When $d \geq 2$, we can prove that (see Appendix A)

$$
\eta_{\ell, m}=\frac{\lambda_{\ell}}{4} \int_{\mathbb{S}^{d}}\left|\mathscr{Y}_{\ell, m}(\xi)\right|^{2} \mathrm{~d} \xi, \forall \ell \geq 0, \forall 1 \leq m \leq \alpha_{\ell} .
$$

When $d=1$, one has

$$
\eta_{\ell, 0}=0, \eta_{\ell, 1}=\frac{\pi}{2} \lambda_{\ell} B_{\ell}^{2}=1, \forall \ell \geq 1 .
$$

We also know that the family $\left(\mathscr{W}_{\ell, m}\right)$ is an orthogonal basis of $\mathbb{W}_{-2}^{0}\left(\mathbb{R}^{d}\right)$ (see [3]). The following proposition is straightforward

Proposition 3.6. If $d \geq 3$, then the solution of Poisson's equation (3.23) is given by

$$
u=\sum_{\ell=0}^{\infty} \sum_{m=1}^{\alpha_{\ell}} \frac{1}{\eta_{\ell, m}}\left\langle f, \mathscr{W}_{\ell, m}\right\rangle \mathscr{W}_{\ell, m} .
$$

Moreover, the discretized problem (3.7) is diagonal and

$$
u_{N}=\sum_{\ell=0}^{N} \sum_{m=1}^{\alpha_{\ell}} \frac{1}{\eta_{\ell, m}}\left\langle f, \mathscr{W}_{\ell, m}\right\rangle \mathscr{W}_{\ell, m} .
$$

When $1 \leq d \leq 2$, the $W_{w}^{1}\left(\mathbb{R}^{d}\right)$-solution of Poisson's equation (3.23) is, up to an additive constant, equal to

$$
u=\sum_{\ell=1}^{\infty} \sum_{m=1}^{\alpha_{\ell}} \frac{1}{\eta_{\ell, m}}\left\langle f, \mathscr{W}_{\ell, m}\right\rangle \mathscr{W}_{\ell, m},
$$

and solution of the discretized problem (3.9) is

$$
u_{N}=c_{0}^{\star}+\sum_{\ell=1}^{N} \sum_{m=0}^{\alpha_{\ell}} \frac{1}{\eta_{\ell, m}}\left\langle f, \mathscr{W}_{\ell, m}\right\rangle \mathscr{W}_{\ell, m},
$$

where the constant $c_{0}^{\star}$ is chosen such that $u_{N}$ satisfies $(2.11)$. In that case

$$
\mathscr{A}\left(\mathscr{W}_{\ell, m}, \mathscr{W}_{r, s}\right)=\eta_{\ell, m} \delta_{\ell, r} \delta_{m, s},
$$

for $\ell, r \geq 0$ and $1 \leq m \leq \alpha_{\ell}, 1 \leq s \leq \alpha_{s}$ and the matrix of the discret problem (3.9) is diagonal.

Remark 3.7. According to orthogonality property (3.18), Proposition 3.6 can easily be extended to problems of the form

$$
-\Delta u+K\left(|x|^{2}+1\right)^{-2} u=f \text { in } \mathbb{R}^{d}
$$

where $K>0$ is a constant. 


\section{IMPLEMENTATION}

In this section, focus is on the numerical implementation of the method in one and multi-dimensional settings. We first give some details concerning the computation of the stiffness matrix from a practical viewpoint. Secondly, in order to check the convergence of the method, we present some computational results.

\subsection{Computing integrals. The stiffness matrix}

Here, we would clear up the computation of integrals involved in computing the stiffness matrix of the discrete system (4.1). We assume that $f$ satisfies (2.4). Decomposing $u_{N}$ on the functions $\mathscr{W}_{\ell, m}, 0 \leq \ell \leq N$ and $1 \leq m \leq \alpha_{\ell}$, transforms the discretized problem (3.7) into a linear system of the form

$$
A U=B,
$$

where $A$ is a square matrix whose entries are $\mathscr{A}\left(\mathscr{W}_{\ell, m}, \mathscr{W}_{j, s}\right)$ or $\mathscr{A}_{\star}\left(\mathscr{W}_{\ell, m}, \mathscr{W}_{j, s}\right), 0 \leq \ell, j \leq N$ and $1 \leq m \leq \alpha_{\ell}$, $1 \leq s \leq \alpha_{j}$. The entries of the right hand side are $\left\langle f, \mathscr{W}_{j, s}\right\rangle$. When $a_{i, j}=\delta_{i, j}$ (Poisson's equation) the matrix of the discrete problem 3.7 is diagonal as explained in Section 3.4.

We are now going to expose a way in which the integrals arising in the coefficients $\mathscr{A}\left(\mathscr{W}_{\ell, m}, \mathscr{W}_{j, s}\right)$ and in the RHS are calculated. A natural idea consists in making a change of variables from $\mathbb{R}^{d}$ to $\mathbb{S}_{\star}^{d}$, compactifying in this way the domain of integration. Let us start by examining integrals of the form

$$
\int_{\mathbb{R}^{d}} f(x) \mathscr{W}(x) \mathrm{d} x
$$

which arise in the RHS. The following formula is proved in Appendix A

$$
\int_{\mathbb{R}^{d}}\left(\frac{2}{|x|^{2}+1}\right)^{\theta} f(x) \phi(x) \mathrm{d} x=\int_{\mathbb{S}^{d}}\left(1-\xi_{d+1}\right)^{\theta-2} \hat{f}(\xi) \hat{\phi}(\xi) \mathrm{d} \xi
$$

for $\theta \in \mathbb{R}$. Here $\hat{f}(\xi)=\left(1-\xi_{d+1}\right)^{-(d-2) / 2} f(\pi(\xi))$. At this stage, spherical coordinates could be used. When $d=1$ or $d=2$, the expression of these coordinates is widely known. When $d=3$, we can write

$$
\xi=\xi(\varphi, \theta, \chi)=(\sin \theta \cos \varphi \sin \chi, \sin \theta \sin \varphi \sin \chi, \cos \theta \sin \chi, \cos \chi)
$$

for $\xi \in \mathbb{S}^{3}$, with $0 \leq \varphi<2 \pi, 0 \leq \theta \leq \pi, 0 \leq \chi \leq \pi$ and we have

$$
\int_{\mathbb{S}^{d}}\left(1-\xi_{d+1}\right)^{\alpha-2} \hat{f}(\xi) \hat{\varphi}(\xi) \mathrm{d} \xi=\int_{0}^{\pi}\left(\int_{0}^{\pi}\left(\int_{0}^{2 \pi}(1-\cos \chi)^{\alpha-2}(\hat{f} \hat{\varphi})(\xi(\varphi, \theta, \chi)) \sin ^{2} \chi \sin \theta \mathrm{d} \varphi\right) \mathrm{d} \theta\right) \mathrm{d} \chi .
$$

It is therefore possible to use a Gauss-Lobatto or a Gauss-Chebyshev quadrature rule.

However, it should be noted that expression (4.2) can be simplified by means of the Funk-Hecke formula at least for the two following cases:

Case 1. If $f$ is radial, that is

$$
f(x)=F(|x|), x \in \mathbb{R}^{d},
$$

for some function $F$ defined on $\mathbb{R}_{+}$. In that case, define the function

$$
h(t)=(1-t)^{-d / 2-1} F\left(\sqrt{\frac{1+t}{1-t}}\right),-1<t<1 .
$$

In view of (4.2), we have

$$
\int_{\mathbb{R}^{d}} f(x) \phi(x) \mathrm{d} x=\int_{\mathbb{S}^{d}} h\left(\xi_{d+1}\right) \hat{\phi}(\xi) \mathrm{d} \xi .
$$


We can now use the Funk-Hecke formula (see, e.g., [46] p. 29 or [4], Thm. 2.22) which states that for all $Y \in \mathbb{H}_{\ell}^{d}$, $\ell \geq 0$ being an integer, and for all $\eta \in \mathbb{S}^{d}$,

$$
\int_{\mathbb{S}^{d}} g(\eta \cdot \xi) Y(\xi) \mathrm{d} \xi=\left|\mathbb{S}^{d-1}\right|\left(\int_{-1}^{1}\left(1-t^{2}\right)^{d / 2-1} P_{\ell, d+1}(t) g(t) \mathrm{d} t\right) Y(\eta),
$$

for any measurable function $g$ measurable on $(-1,1)$ satisfying

$$
\int_{-1}^{1}\left(1-t^{2}\right)^{d / 2-1}|g(t)| \mathrm{d} t<+\infty
$$

Here $P_{\ell, d+1}$ denotes the Legendre polynomial of degree $\ell$ in $d+1$ dimensions given by

$$
P_{\ell, d+1}(t)=\frac{(-1)^{\ell} \Gamma\left(\frac{d}{2}\right)}{2^{\ell} \Gamma\left(\ell+\frac{d}{2}\right)}\left(1-t^{2}\right)^{1-d / 2} \frac{\mathrm{d}^{\ell}}{\mathrm{d} t^{\ell}}\left(1-t^{2}\right)^{\ell+d / 2-1} .
$$

We get

$$
\int_{\mathbb{R}^{d}} f(x) \phi(x) \mathrm{d} x=\left|\mathbb{S}^{d-1}\right|\left(\int_{-1}^{1}\left(1-t^{2}\right)^{d / 2-1} P_{\ell, d+1}(t) h(t) \mathrm{d} t\right) \hat{\phi}\left(\boldsymbol{e}_{d+1}\right),
$$

where $\boldsymbol{e}_{d+1}$ is the $(d+1)$ th canonical vector of $\mathbb{R}^{d+1}$. The integral on the right hand side can be computed by means of a Gauss-Jacobi quadrature formula (see, e.g., [47], Chap. 4).

It is worth noting that formula (4.5) can be simplified when $h \in \mathscr{C}^{\ell}([-1,1])$ and writes

$$
\int_{-1}^{1}\left(1-t^{2}\right)^{d / 2-1} P_{\ell, d+1}(t) h(t) \mathrm{d} t=\frac{\Gamma\left(\frac{d}{2}\right)}{2^{\ell} \Gamma\left(\ell+\frac{d}{2}\right)} \int_{-1}^{1}\left(1-t^{2}\right)^{\ell+d / 2-1} h^{(\ell)}(t) \mathrm{d} t .
$$

Case 2. In this second case, $f$ is of the form

$$
f(x)=\left(\frac{2}{|x|^{2}+1}\right)^{d / 2+1} F\left(\frac{2 x \cdot \boldsymbol{u}}{|x|^{2}+1}\right),
$$

for some unit vector $\boldsymbol{u}=\left(u_{1}, \ldots, u_{d}\right) \in \mathbb{S}^{d-1}$. Using (4.2) gives

$$
\int_{\mathbb{R}^{d}} f(x) \phi(x) \mathrm{d} x=\int_{\mathbb{S}^{d}} F(\xi \cdot \boldsymbol{u}) \hat{\phi}(\xi) \mathrm{d} \xi,
$$

where we still denote by $\boldsymbol{u}$ the vector $\left(u_{1}, \ldots, u_{d}, 0\right) \in \mathbb{S}^{d}$. By the Funk-Hecke formula (4.3) we get

$$
\int_{\mathbb{R}^{d}} f(x) \phi(x) \mathrm{d} x=\left|\mathbb{S}^{d-1}\right|\left(\int_{-1}^{1}\left(1-t^{2}\right)^{d / 2-1} P_{\ell, d+1}(t) F(t) \mathrm{d} t\right) \hat{\phi}(\boldsymbol{u}) .
$$

Remark 4.1. The polynomial $P_{\ell, d+1}$ is proportional to Jacobi polynomial of degree $\ell, J_{\ell}^{(\alpha, \alpha)}$ with $\alpha=d / 2-1$. Moreover, $P_{\ell, 2}$ is the ordinary Chebyshev polynomial of degree $\ell, P_{\ell, 3}$ is the Legendre polynomial of degree $\ell$ and $P_{\ell, 4}=1 /(\ell+1) U_{\ell}$, where $U_{\ell}$ is the $\ell$-degree Chebyshev polynomial of the second kind.

Now, let us come back to the calculation of the entries $\mathscr{A}\left(\mathscr{W}_{\ell, m}, \mathscr{W}_{j, s}\right)$. In the one dimensional case $(d=1)$, a direct change of variables based on formula (3.16) appears to be appropriated. More precisely, set

$$
h(x)=\left(x^{2}+1\right) f(x), x \in \mathbb{R}, \tilde{b}(x)=\sqrt{x^{2}+1} b(x), \tilde{c}(x)=\left(x^{2}+1\right) c(x), x \in \mathbb{R} .
$$

According to Assumptions (2.10), we get

$$
\int_{\mathbb{R}} \frac{|h(x)|^{2}}{x^{2}+1} \mathrm{~d} x<+\infty, \tilde{b} \in L^{\infty}(\mathbb{R}), \tilde{c} \in L^{\infty}(\mathbb{R}) .
$$


Then

$$
\begin{aligned}
\mathscr{A}\left(\mathscr{W}_{\ell, 1}, \mathscr{W}_{j, 1}\right)= & B_{\ell} B_{j}\left(\int_{-1}^{1} a\left(\frac{t}{\sqrt{1-t^{2}}}\right) U_{\ell}^{\prime}(t) U_{j}^{\prime}(t)\left(1-t^{2}\right)^{3 / 2} \mathrm{~d} t\right. \\
& +\int_{-1}^{1} \tilde{b}\left(\frac{t}{\sqrt{1-t^{2}}}\right) U_{\ell}^{\prime}(t) U_{j}(t)\left(1-t^{2}\right)^{1 / 2} \mathrm{~d} t \\
& \left.+\int_{-1}^{1} \tilde{c}\left(\frac{t}{\sqrt{1-t^{2}}}\right) U_{\ell}(t) U_{j}(t)\left(1-t^{2}\right)^{-1 / 2} \mathrm{~d} t\right) .
\end{aligned}
$$

In the particular case $a=1, b=c=0$, one has

$$
\mathscr{A}\left(\mathscr{W}_{\ell, 1}, \mathscr{W}_{j, 1}\right)=\delta_{\ell, j}
$$

and the matrix of the linear system (3.7) is the identity. In the more general situation in which $a$ is a variable function, integrals $\mathscr{A}\left(\mathscr{W}_{\ell, 1}, \mathscr{W}_{j, 1}\right)$ can be computed with help of Chebyshev-Gauss quadrature formula

$$
\int_{-1}^{1}\left(1-t^{2}\right)^{-1 / 2} g(t) \mathrm{d} t \approx \sum_{i=1}^{N^{\star}} \varrho_{i} g\left(\cos \left(\theta_{i}\right)\right)
$$

where $N^{\star} \geq 1$ is the number of nodes and

$$
\theta_{i}=\frac{2 i-1}{2 N^{\star}} \pi, \varrho_{i}=\frac{\pi}{N^{\star}}, 1 \leq i \leq N^{\star} .
$$

It is worth recalling the formula

$$
\begin{aligned}
(\sin \theta) U_{\ell}(\cos (\theta)) & =\sin ((\ell+1) \theta), \\
(\sin \theta)^{3} U_{\ell}^{\prime}(\cos (\theta)) & =\sin ((\ell+1) \theta) \cos (\theta)-(\ell+1) \cos ((\ell+1) \theta) \sin \theta .
\end{aligned}
$$

Similarly,

$$
\int_{\mathbb{R}} f(x) \mathscr{W}_{\ell, 1}(x) \mathrm{d} x=B_{\ell} \int_{-1}^{1} h\left(\frac{t}{\sqrt{1-t^{2}}}\right) U_{\ell}(t)\left(1-t^{2}\right)^{-1 / 2} \mathrm{~d} t .
$$

This integral can be numerically computed by means of formula (4.9). When $b=0$ and $c=0$, we also need the formula

$$
\int_{\mathbb{R}} \frac{\mathscr{W}_{\ell, 1}(x)}{x^{2}+1} \mathrm{~d} x=B_{\ell} \int_{-1}^{1}\left(1-t^{2}\right)^{-1 / 2} U_{\ell}(t) \mathrm{d} t
$$

In the multi-dimensional case $(d \geq 1)$, the computation of the integrals arising in the entries $\mathscr{A}\left(\mathscr{W}_{\ell, m}, \mathscr{W}_{j, s}\right)$ can be done by means of a change of variables from $\mathbb{R}^{d}$ to $\mathbb{S}_{\star}^{d}$, as explained above. In order to describe in detail the methodology, let us assume for simplicity that $\boldsymbol{b}=0$ and $c=0$ (the case $\boldsymbol{b} \neq 0$ or/and $c \neq 0$ can be treated in a similar way). We stated earlier that the arising matrix is diagonal for Poisson's equation (3.23). In the more general situation, we prove the formula (see Appendix A for the proof)

$$
\mathscr{A}(u, v)=\int_{\mathbb{S}^{d}} \frac{\left[\nabla_{\xi} \widehat{u}(\xi)^{t} H(\xi)^{t}-\alpha \widehat{u}(\xi) \xi_{*}^{t}\right] \widehat{A}(\xi)\left[H(\xi) \nabla_{\xi} \widehat{v}(\xi)-\alpha \widehat{v}(\xi) \xi_{*}\right]}{\left(1-\xi_{d+1}\right)^{2}} \mathrm{~d} \xi
$$

where

$$
\widehat{u}(\xi)=\left(1-\xi_{d+1}\right)^{-\alpha} u(\pi(\xi)), \text { for } \xi \in \mathbb{S}_{\star}^{d}, \text { with } \alpha=(d-2) / 2,
$$

( $\widehat{v}$ is similarly defined). Here $H(\xi)$ denotes the matrix defined by (A.7) (see Appendix A). The integral on the right hand side of (4.12) is absolutely convergent and can be directly computed using a quadrature formula on the unit sphere (see, e.g., $[1,27,41,44])$. Similar formulae can be easily obtained when $\boldsymbol{b} \neq 0$ or $c \neq 0$.

Of course, other changes of variables can be used (see the forthcoming paper [14] for more details). 


\subsection{Numerical results}

We are now in position to provide a quantitative assessment of the method through some computational tests. The method is implemented in the one dimensional case $(d=1)$ for solving problems of the form

$$
-\left(a u^{\prime}\right)^{\prime}(x)+b(x) u^{\prime}(x)+c(x) u(x)=f(x), x \in \mathbb{R} .
$$

completed with the integral condition (2.11). It is also implemented for the three dimensional Poisson's equation $(d=3)$

$$
-\Delta u=f \text { in } \mathbb{R}^{3} .
$$

In all the cases, we observe the behavior of the relatives errors

$$
e_{0}=\frac{\left\|u-u_{N}\right\|_{\mathbb{W}_{-1}^{0}\left(\mathbb{R}^{d}\right)}}{\|u\|_{\mathbb{W}_{-1}^{0}\left(\mathbb{R}^{d}\right)}}, e_{1}=\frac{\left\|\nabla\left(u-u_{N}\right)\right\|_{L^{2}\left(\mathbb{R}^{d}\right)}}{\|\nabla u\|_{L^{2}\left(\mathbb{R}^{d}\right)}},
$$

and we give a comparison with error estimates established hereabove. Four examples are considered. In all these examples, quadrature formulae, such as (4.9), are used with $N^{\star}=20 N$. Such a choice ensures that the numerical integration does not have a significant effect on the error of approximation.

Example 4.2. First, we consider an example with a smooth solution of the 1-dimensional equation (4.13), considered when $a=1, b=c=0$, and completed with the condition

$$
\int_{\mathbb{R}} \frac{u(x)}{x^{2}+1} \mathrm{~d} x=0
$$

The right-hand side is chosen such that the solution is

$$
u(x)=\frac{x}{\left(x^{2}+1\right)^{\frac{s}{2}+\frac{1}{4}} \log \left(x^{2}+2\right)},
$$

for some real number $s$. The behavior of $u$ at larges distances depends on $s$. More precisely, for each $m \geq 0$, there exists a constant $C_{m}>0$ such that

$$
\left|u^{(m)}(x)\right| \leq C_{m} \frac{\langle x\rangle^{\frac{1}{2}-s-m}}{\log \left(x^{2}+2\right)}, \text { for all } x \in \mathbb{R} .
$$

It follows that $u \in \mathbb{W}_{2 s}^{s+1}(\mathbb{R})$. Moreover, $u \notin \mathbb{W}_{2 r}^{r+1}(\mathbb{R})$ for any real $r>s$. According to Theorem 3.2, we expect that the error in $W_{w}^{1}$ norm decreases at least as $N^{-s}$.

In Table 1, the relative errors $e_{0}$ and $e_{1}$ are illustrated for several choices of $s$ and $N$. The log-plots of these errors are given in Figures 1 and 2 when $s=1,2$ or 3 . As expected the convergence to the exact solution is of algebraic type and it is achieved with an index approximately equal to $s$. This is obviously in agreement with statements of Theorem 3.2.

On the other hand, it is worth noting that the method is still convergent when $u$ increases slowly when $|x| \rightarrow \infty$. Figure 3 compares the exact solution with the approximate one when $s=1 / 5$ and $N=40$. The solutions are indistinguishable to within the thickness of the curves, despite the fact that the exact solution increases as $|x|^{3 / 10}(\log |x|)^{-1}$ when $|x| \rightarrow \infty$.

Example 4.3. In this second example, we consider equation (4.13) when the coefficient $a$ oscillates in near and far regions;

$$
a(x)=1+\frac{1}{2} \cos (2 x), x \in \mathbb{R},
$$




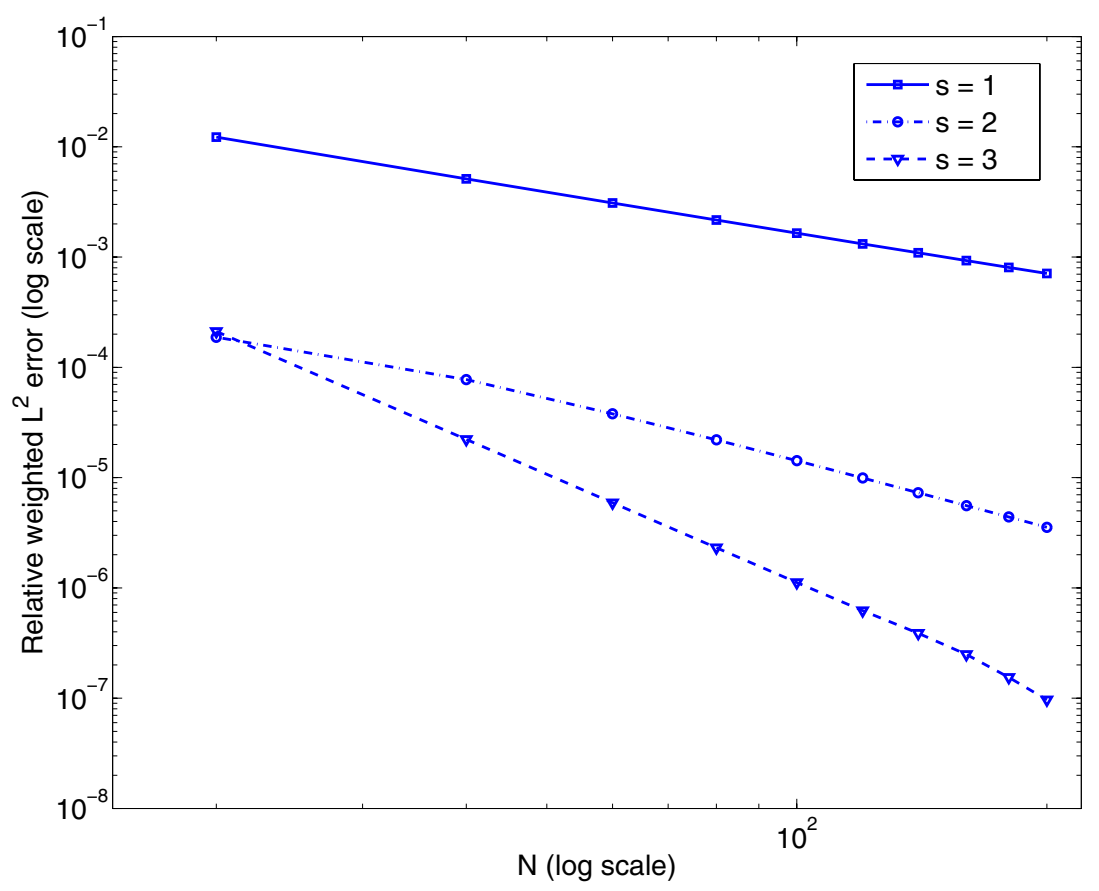

Figure 1. (Example 4.2) Relative weighted error $e_{0}$ versus $N$ for several values of $s$ (in log scale).

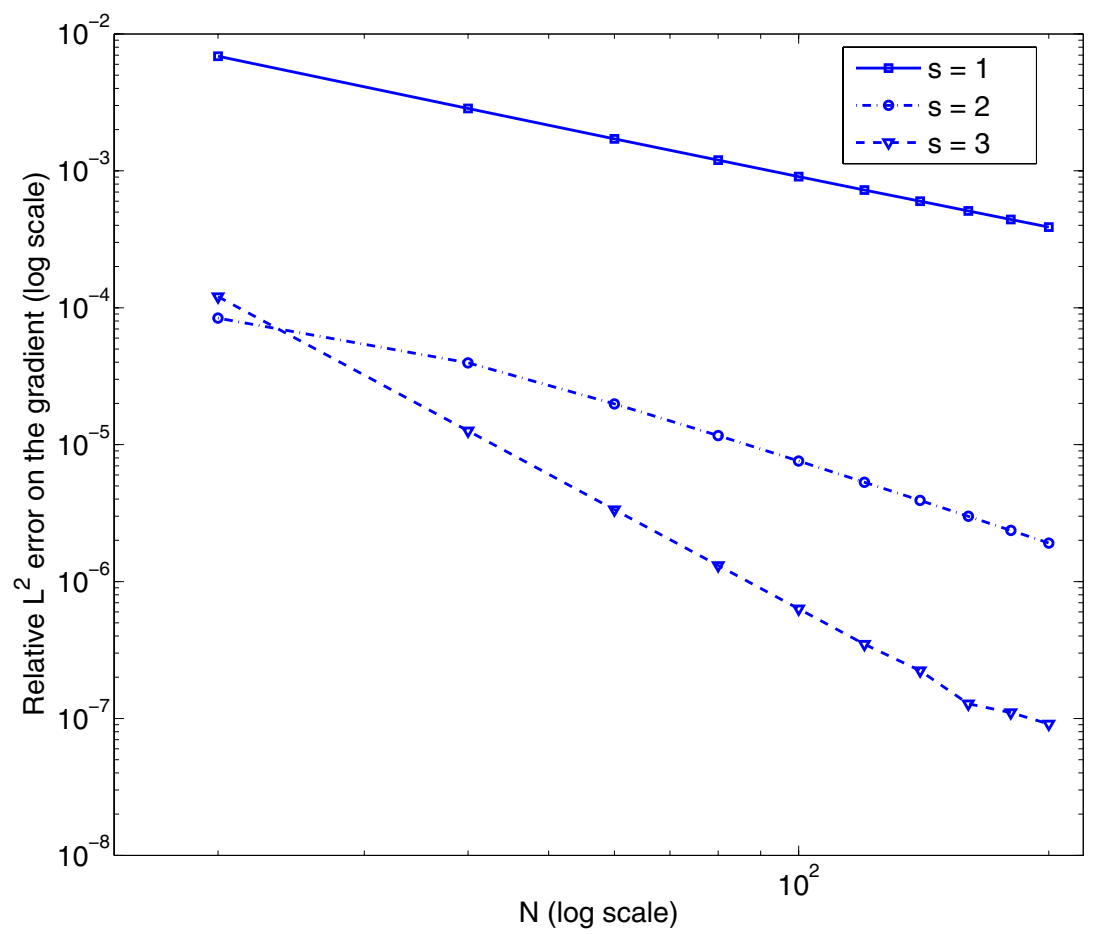

Figure 2. (Example 4.2) Relative weighted error $e_{1}$ versus $N$ for several values of $s$ (in log scale). 
TABLE 1. Relative errors $e_{0}$ and $e_{1}$ obtained for Example 4.1 for several values of the parameter $s$ and the discretization parameter $N$.

\begin{tabular}{ccccccc}
\hline & \multicolumn{2}{c}{$s=1$} & \multicolumn{2}{c}{$s=2$} & \multicolumn{2}{c}{$s=3$} \\
\hline$N$ & $e_{0}$ & $e_{1}$ & $e_{0}$ & $e_{1}$ & $e_{0}$ & $e_{1}$ \\
\hline 20 & $1.223 \mathrm{e}-2$ & $6.875 \mathrm{e}-3$ & $1.874 \mathrm{e}-4$ & $8.403 \mathrm{e}-5$ & $2.118 \mathrm{e}-4$ & $1.206 \mathrm{e}-4$ \\
40 & $5.121 \mathrm{e}-3$ & $2.854 \mathrm{e}-3$ & $7.754 \mathrm{e}-5$ & $3.964 \mathrm{e}-5$ & $2.223 \mathrm{e}-5$ & $1.263 \mathrm{e}-5$ \\
60 & $3.091 \mathrm{e}-3$ & $1.713 \mathrm{e}-3$ & $3.787 \mathrm{e}-5$ & $1.987 \mathrm{e}-5$ & $5.919 \mathrm{e}-6$ & $3.353 \mathrm{e}-6$ \\
80 & $2.166 \mathrm{e}-3$ & $1.197 \mathrm{e}-3$ & $2.199 \mathrm{e}-5$ & $1.166 \mathrm{e}-5$ & $2.316 \mathrm{e}-6$ & $1.311 \mathrm{e}-6$ \\
100 & $1.647 \mathrm{e}-3$ & $9.075 \mathrm{e}-4$ & $1.424 \mathrm{e}-5$ & $7.594 \mathrm{e}-6$ & $1.119 \mathrm{e}-6$ & $6.324 \mathrm{e}-7$ \\
120 & $1.318 \mathrm{e}-3$ & $7.249 \mathrm{e}-4$ & $9.93 \mathrm{e}-6$ & $5.31 \mathrm{e}-6$ & $6.218 \mathrm{e}-7$ & $3.49 \mathrm{e}-7$ \\
140 & $1.093 \mathrm{e}-3$ & $6.000 \mathrm{e}-4$ & $7.296 \mathrm{e}-6$ & $3.912 \mathrm{e}-6$ & $3.885 \mathrm{e}-7$ & $2.233 \mathrm{e}-7$ \\
160 & $9.295 \mathrm{e}-4$ & $5.097 \mathrm{e}-4$ & $5.575 \mathrm{e}-6$ & $2.995 \mathrm{e}-6$ & $2.497 \mathrm{e}-7$ & $1.279 \mathrm{e}-7$ \\
180 & $8.062 \mathrm{e}-4$ & $4.416 \mathrm{e}-4$ & $4.391 \mathrm{e}-6$ & $2.363 \mathrm{e}-6$ & $1.544 \mathrm{e}-7$ & $1.105 \mathrm{e}-7$ \\
200 & $7.101 \mathrm{e}-4$ & $3.886 \mathrm{e}-4$ & $3.544 \mathrm{e}-6$ & $1.910 \mathrm{e}-6$ & $9.662 \mathrm{e}-8$ & $9.118 \mathrm{e}-8$ \\
\hline Log slope (approximate) & -1.23 & -1.24 & -2.00 & -1.99 & -3.30 & -3.15 \\
\hline
\end{tabular}

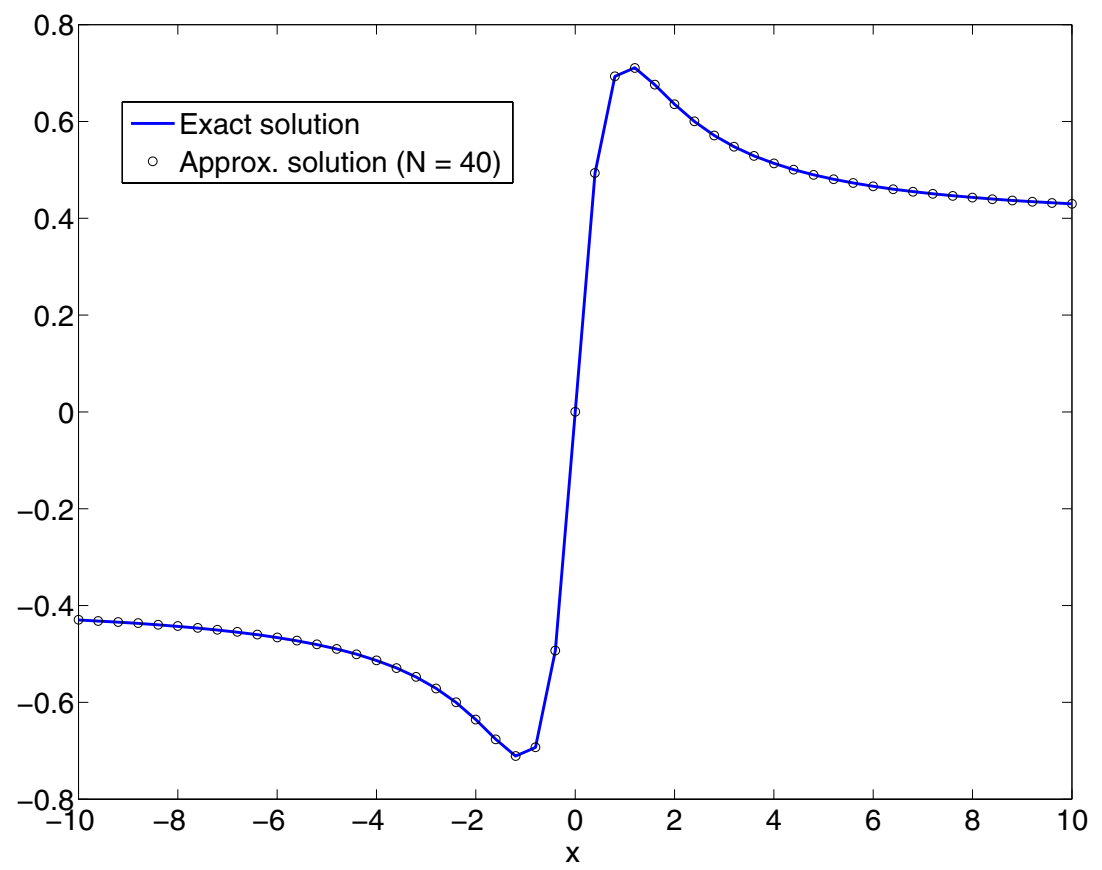

Figure 3. The approximation and the exact solution for Example 4.2 when $s=\frac{1}{5}$ and $N=40$.

$(b=c=0)$. The source term $f$ is chosen to be consistent with the exact solution

$$
u(x)=\frac{\sin x}{\left(x^{2}+1\right)^{3 / 2}} .
$$

Here also, the approximate solution is in good agreement with the exact one as it is shown in Figure 4. Both the relative weighted $L^{2}$ error $e_{0}$ and the relative $L^{2}$ error on the gradient $e_{1}$ decrease approximately as $N^{-1}$ (see Figs. 5 and 6). This is in line with forecasts of Theorem 3.3 since $u \in \mathbb{W}_{2}^{2}(\mathbb{R})$ but $u \notin \mathbb{W}_{2 r}^{r+1}(\mathbb{R})$ for $r \geq 5 / 4$. 


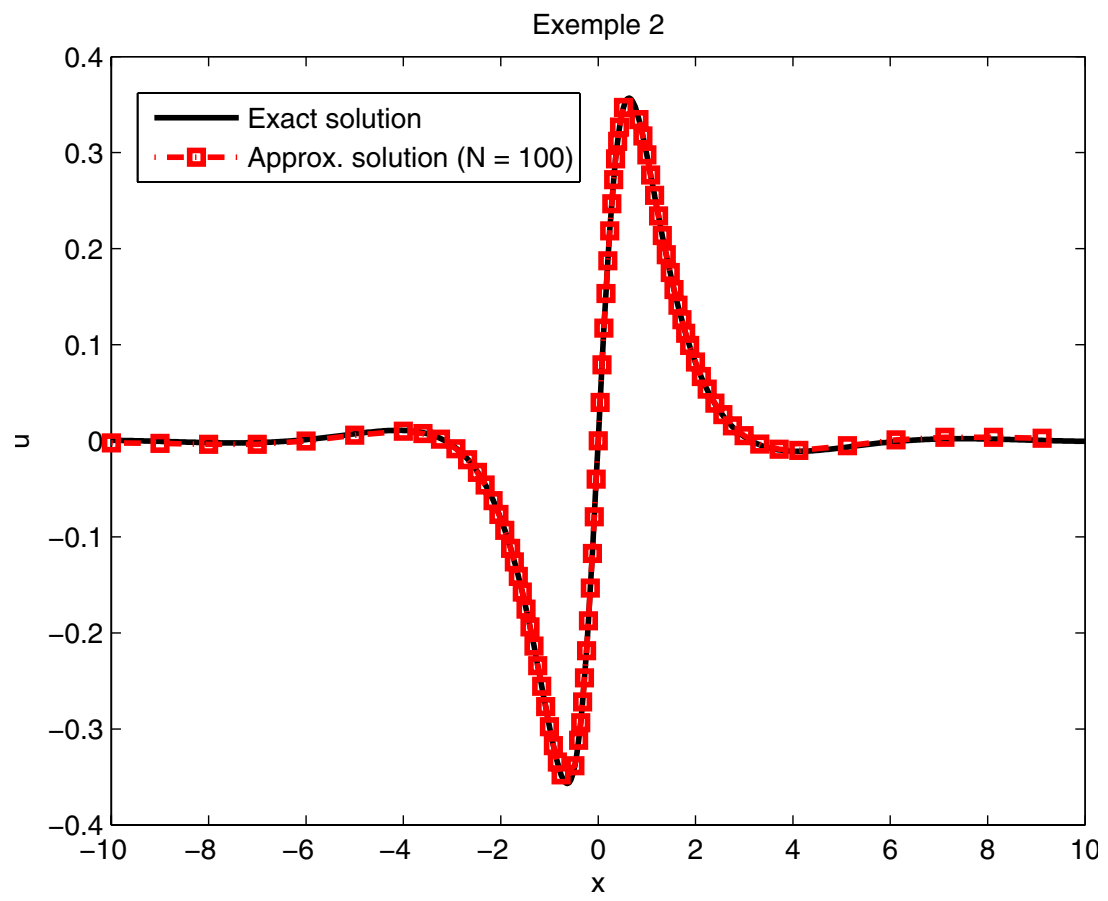

Figure 4. The approximate solution and the exact one for the second example.

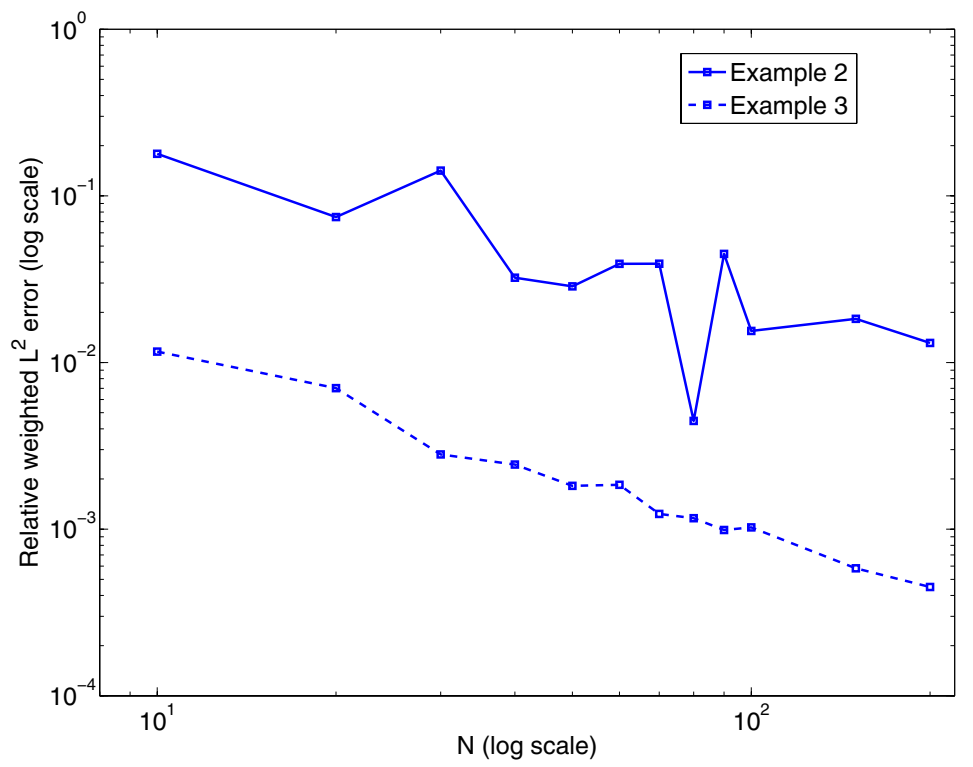

FiguRE 5. Relative weighted $L^{2}$ error for Example 4.3 (solid) and Example 4.4 (dashed) versus $N$ (in $\log$ scale). 


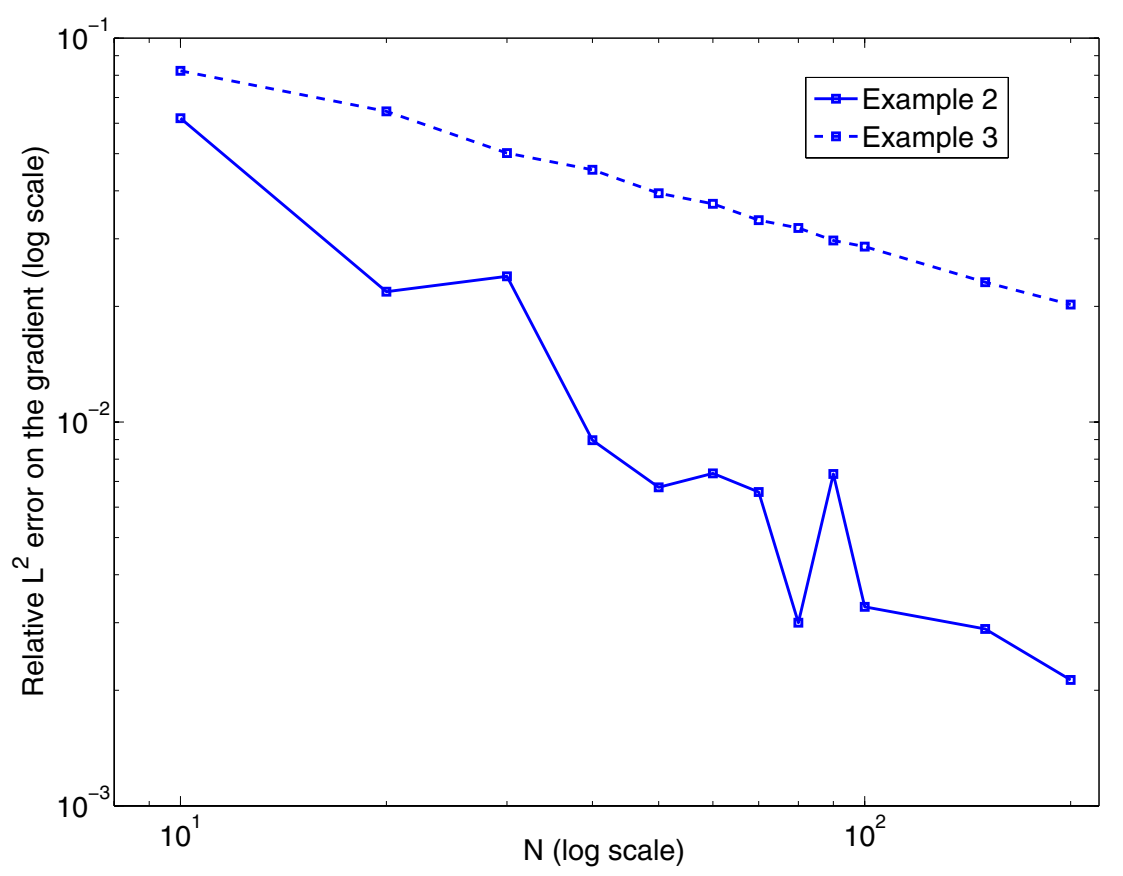

Figure 6. Relative $L^{2}$ error on the gradients for Example 4.3 (solid) and Example 4.4 (dashed) versus $N$ (in log scale).

Example 4.4. In this example, equation (4.13) is considered with a discontinuous coefficient

$$
a(x)= \begin{cases}1 & \text { if }|x| \leq 1 \\ a_{0} & \text { if }|x|>1\end{cases}
$$

where $a_{0}>0$ is a constant $(b=c=0)$. The source term is given by

$$
f(x)=3 \frac{x}{\left(1+x^{2}\right)^{5 / 2}} .
$$

The unique solution of (4.13) satisfying (2.11) is

$$
u(x)=a(x)^{-1} u_{0}(x)+u_{1}(x) \text { for } x \in \mathbb{R},|x| \neq 1,
$$

where

$$
u_{0}(x)=\frac{x}{\left(x^{2}+1\right)^{1 / 2}} .
$$

while $u_{1}$ is a piecewise linear function chosen such that $u \in W_{w}^{1}(\mathbb{R})$ and satisfies (4.13). Since $u$ and $a u^{\prime}$ must be continuous at \pm 1 , one can prove that

$$
u_{1}(x)= \begin{cases}0 & \text { if }|x| \leq 1 \\ k_{0} & \text { if } x>1 \\ -k_{0} & \text { if } x<-1\end{cases}
$$

with $k_{0}=\left(1-a_{0}^{-1}\right) u_{0}(1)$. One can notice, on one hand, that the solution $u$ is not very smooth since it does not belong to $H_{l o c}^{2}(\mathbb{R})$. On the other hand, $|u(x)|$ does not decrease to zero when $|x| \rightarrow+\infty$. More precisely, $u(x) \rightarrow \pm u_{0}(1) \pm\left(1-u_{0}(1)\right) a_{0}^{-1}$ when $x \rightarrow \pm \infty$. 


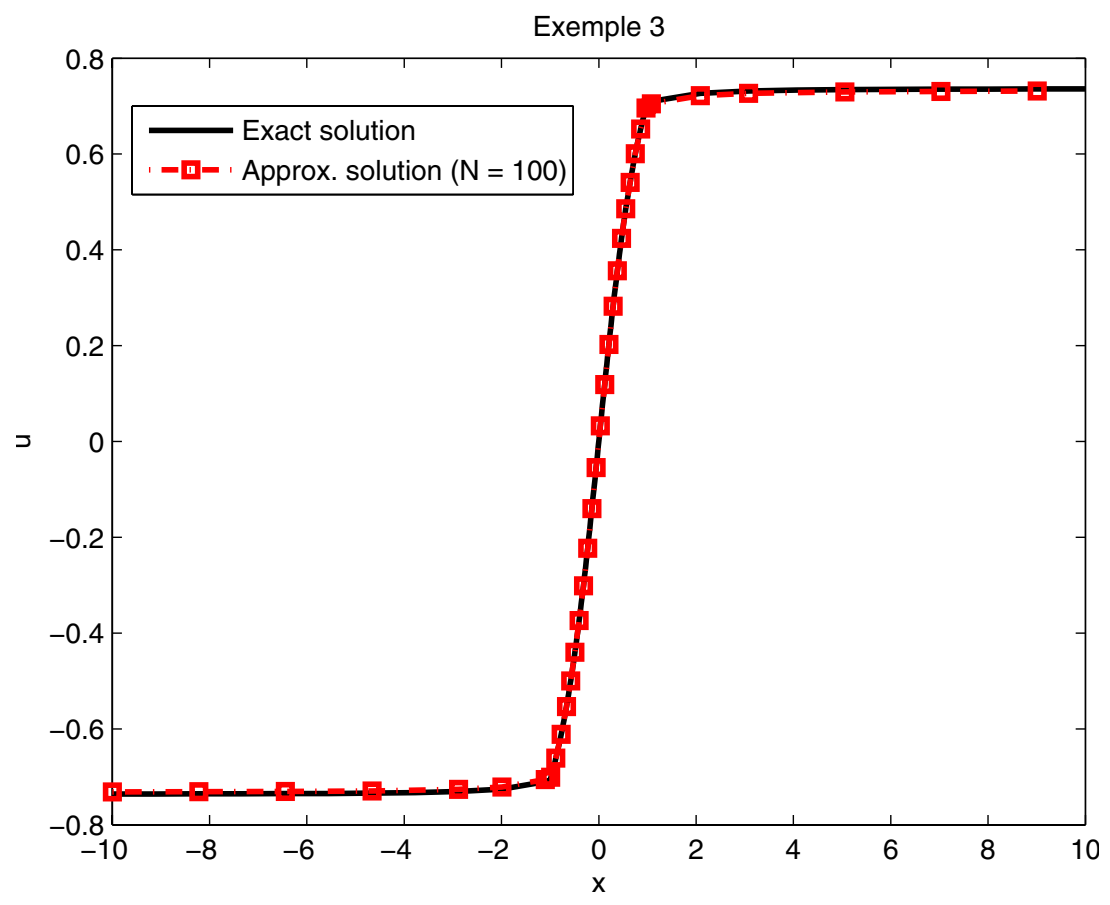

FiguRE 7. The approximate and the exact solution for Example 4.4.

In Figure 7, we display the exact and approximate solutions when $a_{0}=10$ and $N=100$. Errors on $u$ and on its gradient are shown in Figures 5 and 6. One can notice that the weighted $L^{2}$ error decreases as $N^{-1.15}$. However, the $L^{2}$-error on the gradient decreases as $\sqrt{N}$. This fact is not inconsistent with the predictions of Theorem 3.2 since the solution is not sufficiently smooth nor quickly decreasing when $|x| \rightarrow+\infty$.

Example 4.5. In this last example, we deal with a three dimensional example. More precisely, we consider Poisson's equation (3.23) with $f$ chosen such that

$$
u(x)=\left(\frac{2}{|x|^{2}+1}\right)^{\frac{3}{2}}\left|x_{1}\right| \operatorname{atan}\left(\frac{2 x_{1}}{|x|^{2}+1}\right) .
$$

Let us recall that for the Poisson equation, the arising system is diagonal. This solution belongs to $W_{2}^{2}\left(\mathbb{R}^{3}\right)$ (but it no longer belongs to $W_{4}^{3}\left(\mathbb{R}^{3}\right)$ nor to $\left.H_{\text {loc }}^{3}\left(\mathbb{R}^{3}\right)\right)$. According to Theorem 3.2 , we expect that the $W_{0}^{1}\left(\mathbb{R}^{d}\right)$-error decreases at least as $N^{-1}$. The results of the numerical tests are presented in Table 2 and in Figure 8. The numerical errors confirm predictions of our error estimate as pointed out in Table 2.

A complementary series of tests concerning Poisson's equation in $\mathbb{R}^{3}$ confirm the efficiency of the method and its fast convergence, particularly for smooth and quickly decreasing solutions.

Overall, these numerical results demonstrate the capabilities of the method we propose here to compute solutions in unbounded geometries. They also point out that the method is accurate far away from the origin. The method can easily be applied to several kinds of partial differential equations and in arbitrary dimensions. In particular, it allows to tackle equations with varying coefficients at infinity. It also avoids any truncation of the domain, preserving by the way its unboundedness. The authors are considering to develop other applications of the method in forthcoming papers (see, e.g., [14]). 
TABLE 2. Relative $W_{0}^{1}$-error and relative $L^{2}$ on the gradient versus $N$ for Example 4.4.

\begin{tabular}{ccc}
\hline$N$ & $e_{0}$ & $e_{1}$ \\
\hline 5 & $3.255 \mathrm{e}-2$ & $9.985 \mathrm{e}-2$ \\
10 & $1.012 \mathrm{e}-2$ & $4.554 \mathrm{e}-2$ \\
15 & $3.496 \mathrm{e}-3$ & $2.985 \mathrm{e}-2$ \\
20 & $2.104 \mathrm{e}-4$ & $1.652 \mathrm{e}-2$ \\
25 & $1.154 \mathrm{e}-4$ & $1.100 \mathrm{e}-2$ \\
30 & $8.32 \mathrm{e}-4$ & $9.221 \mathrm{e}-3$ \\
35 & $5.46 \mathrm{e}-4$ & $6.989 \mathrm{e}-3$ \\
40 & $4.274 \mathrm{e}-4$ & $5.857 \mathrm{e}-3$ \\
\hline Log slope & -2.08 & -1.36 \\
\hline \multicolumn{3}{c}{}
\end{tabular}

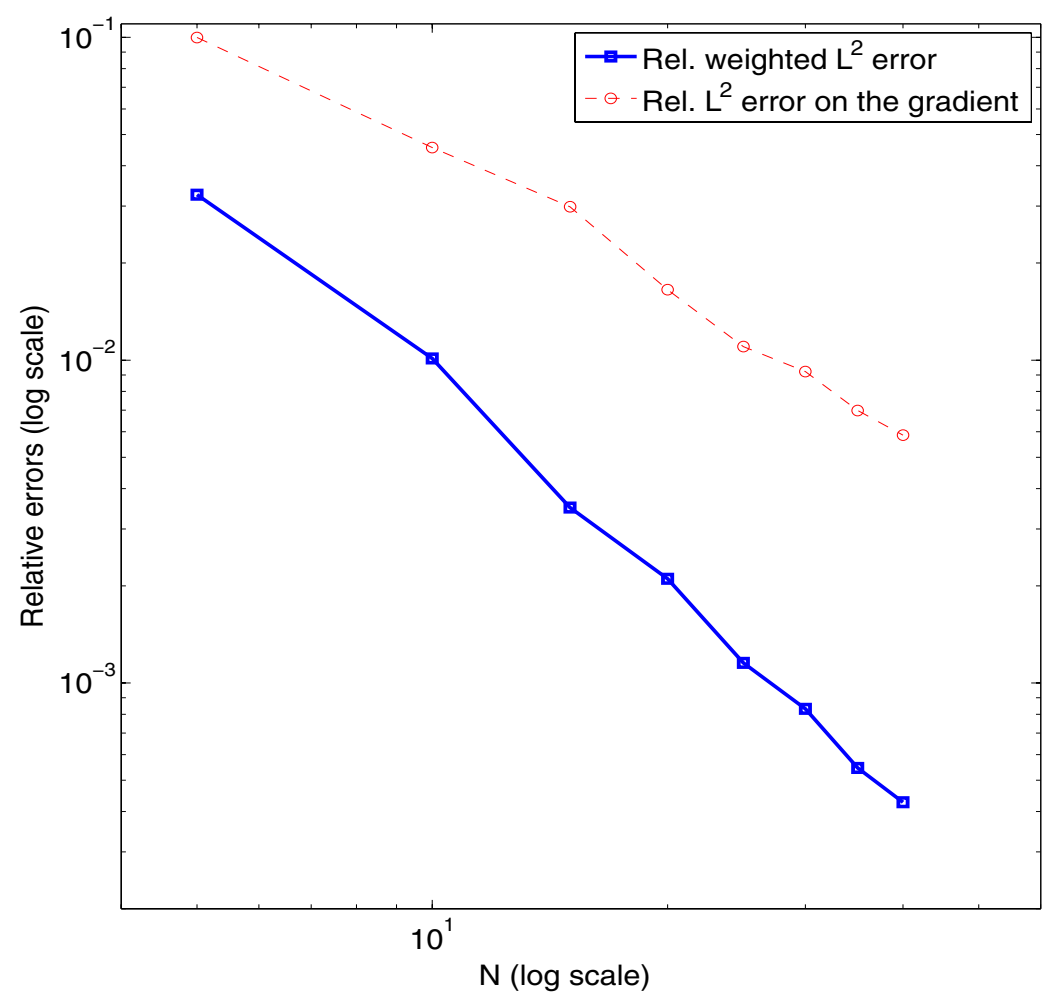

Figure 8. (Example 4.4) Relative weighted errors $e_{0}$ and $e_{1}$ versus $N$ (in log scale).

\section{Appendix A. The Stereographic PROJection AND Integrals}

In what follows, given a function $h$ in $\mathscr{D}\left(\mathbb{R}^{d}\right)$ (the space of infinitely differentiable functions with compact support), we denote by $\widetilde{h}$ the function defined over $\mathbb{S}_{\star}^{d}$ by

$$
\widetilde{h}(\xi)=h(\pi(\xi)), \text { for } \xi \in \mathbb{S}_{\star}^{d} .
$$

Let $B^{\star \star}=B-\{(0, \ldots, 0, t), 0 \leq t<1\}$, where $B$ is the unit ball of $\mathbb{R}^{d+1}$. Then,

$$
\int_{\mathbb{S}^{d}} \widetilde{h}(\xi) \mathrm{d} \xi=(d+1) \int_{B^{\star \star}} \widetilde{F}(x) \mathrm{d} x
$$


where $\widetilde{F}$ is the degree zero homogeneous extension of $\widetilde{h}$ to $B^{\star \star}$ defined by

$$
\forall x \in B^{\star \star}, \widetilde{F}(x)=\widetilde{h}\left(\frac{x}{|x|}\right)
$$

Consider now the one-to-one map

$$
\begin{aligned}
\varphi: B^{\star \star} & \left.\longrightarrow \mathbb{R}^{d} \times\right] 0,1[ \\
x & \longmapsto(y, r)=(\pi(\xi), r) \text { where } \xi=\frac{x}{|x|}, r=|x| .
\end{aligned}
$$

We have

$$
\int_{B^{\star \star}} \widetilde{F}(x) \mathrm{d} x=\int_{\left.\mathbb{R}^{d} \times\right] 0,1[} \widetilde{F}\left(\varphi^{-1}(y, r)\right)\left|J_{\varphi}(y, r)\right|^{-1} \mathrm{~d} y \mathrm{~d} r,
$$

where $J_{\varphi}(y, r)$ is the jacobian matrix of this variable changement. One has

$$
J_{\varphi}(y, r)=-\left(\frac{1+|y|^{2}}{2 r}\right)^{d}
$$

It follows that

$$
\frac{1}{d+1} \int_{\mathbb{S}^{d}} \widetilde{h}(\xi) \mathrm{d} \xi=\int_{B^{\star \star}} \widetilde{F}(x) \mathrm{d} x=\frac{1}{d+1} \int_{\mathbb{R}^{d}} h(y)\left(\frac{2}{1+|y|^{2}}\right)^{d} \mathrm{~d} y .
$$

We get the formula

$$
\int_{\mathbb{R}^{d}} h(y)\left(\frac{2}{1+|y|^{2}}\right)^{d} \mathrm{~d} y=\int_{\mathbb{S}^{d}} \widetilde{h}(\xi) \mathrm{d} \xi .
$$

Formulae (3.25) and (4.2) are direct consequences of (A.2).

On the other hand, since $h(y)=\widetilde{F} \circ \pi^{-1}(y)$ for all $y \in \mathbb{R}^{d}$, one has

$$
\nabla h(y)=M(\xi) \nabla_{x} \widetilde{F}(\xi)=M(\xi) \cdot \nabla_{\xi} \widetilde{h}(\xi), y \in \mathbb{R}^{d}, \xi=\pi^{-1}(y),
$$

where $\nabla_{\xi} \widetilde{h}$ is the tangential gradient of $\widetilde{h}$. The entries of the rectangular matrix $M(\xi)$ are given by

$$
\begin{aligned}
m_{i, j}(\xi) & =\left(1-\xi_{d+1}\right) \delta_{i, j}-\xi_{i} \xi_{j} \text { if } 1 \leq i \leq d, 1 \leq j \leq d \\
m_{i, d+1}(\xi) & =\left(1-\xi_{d+1}\right) \xi_{i} .
\end{aligned}
$$

One can easily prove this

$$
\begin{aligned}
& M(\xi) M(\xi)^{t}=\left(1-\xi_{d+1}\right)^{2} I_{d}, \\
& M(\xi)^{t} M(\xi)=\left(1-\xi_{d+1}\right)^{2}\left(I_{d+1}-\xi \xi^{t}\right), \text { for } \xi \in \mathbb{S}_{\star}^{d} .
\end{aligned}
$$

Furthermore, for any tangential vector $z \in \mathbb{R}^{d+1}$ (that is, $\xi^{t} . z=0$ ), one has

$$
M(\xi) z=\left(1-\xi_{d+1}\right) z_{*}+z_{d+1} \xi_{*}, \xi_{*}^{t} M(\xi) z=\left(1-\xi_{d+1}\right) z_{d+1}
$$

where $z_{*}=\left(z_{1}, \ldots, z_{d}\right)^{t}$ and $\xi_{*}=\left(\xi_{1}, \ldots, \xi_{d}\right)^{t}$. In other words, (A.3) can be rewritten into the form

$$
\widetilde{\nabla_{x} h}(\xi)=H(\xi) \nabla_{\xi} \widetilde{h}(\xi)
$$

where $H(\xi)=\left(h_{i, j}(\xi)\right)_{1 \leq i \leq d, 1 \leq j \leq d+1}$ is a $d \times(d+1)$ is given by

$$
\begin{aligned}
h_{i, j}(\xi) & =\left(1-\xi_{d+1}\right) \delta_{i, j} \text { if } 1 \leq i \leq d, 1 \leq j \leq d, \\
h_{i, d+1}(\xi) & =\xi_{i}
\end{aligned}
$$


Now, given two functions $u$ and $v$ in $\mathscr{D}\left(\mathbb{R}^{d}\right)$, we set

$$
U(x)=\frac{u(x)}{\rho(x)}, V(x)=\frac{v(x)}{\rho(x)} \text { with } \rho(x)=\left(\frac{2}{|x|^{2}+1}\right)^{\frac{d-2}{2}} .
$$

One has $\widetilde{\rho}(\xi)=\left(1-\xi_{d+1}\right)^{\alpha}$ and $\widetilde{\nabla}(\xi)=-\alpha\left(1-\xi_{d+1}\right)^{\alpha} \xi_{*}$, with $\alpha=(d-2) / 2$ and $\xi_{*}=\left(\xi_{1}, \ldots, \xi_{d}\right)^{T}$. It follows that

$$
\widetilde{\nabla u}=\left(1-\xi_{d+1}\right)^{\alpha}\left[H(\xi) \nabla_{\xi} \widetilde{U}-\alpha \widetilde{U}(\xi) \xi_{*}\right]
$$

Using relations (A.2) and (A.9) one can easily get formula (4.12). The formula remains valid when $u$ and $v$ belong to $W_{w}^{1}\left(\mathbb{R}^{d}\right)$. In that case $\widetilde{U}=\widehat{u} \in H^{1}\left(\mathbb{S}_{\star}^{d}\right)$ and $\widetilde{V}=\widehat{v} \in H^{1}\left(\mathbb{S}_{\star}^{d}\right)$ (see [3]).

We can go further into this calculation in the case of Poisson's equation $\left(a_{i, j}=\delta_{i, j}\right.$ for $\left.1 \leq i, j \leq n\right)$. Since $H(\xi)$ can be replaced by $M(\xi)$ in (A.9), we can write

$$
\begin{aligned}
\mathscr{A}(u, v)= & \int_{\mathbb{S}^{d}} \frac{\nabla_{\xi} \widetilde{U}(\xi)^{t} M(\xi)^{t} M(\xi) \nabla_{\xi} \widetilde{V}(\xi)}{\left(1-\xi_{d+1}\right)^{2}} \mathrm{~d} \xi+\alpha^{2} \int_{\mathbb{S}^{d}} \frac{\left|\xi_{*}\right|^{2} \widetilde{U}(\xi) \widetilde{V}(\xi)}{\left(1-\xi_{d+1}\right)^{2}} \mathrm{~d} \xi \\
& -\alpha \int_{\mathbb{S}^{d}} \frac{\xi_{*}^{t} M(\xi) \nabla_{\xi}(\widetilde{U} \widetilde{V})(\xi)}{\left(1-\xi_{d+1}\right)^{2}} \mathrm{~d} \xi \\
= & \int_{\mathbb{S}^{d}} \nabla_{\xi} \widetilde{U}(\xi)^{t} \cdot \nabla_{\xi} \widetilde{V}(\xi)+\alpha^{2} \int_{\mathbb{S}^{d}} \frac{\left(1+\xi_{d+1}\right) \widetilde{U}(\xi) \widetilde{V}(\xi)}{1-\xi_{d+1}} \mathrm{~d} \xi \\
& -\alpha \int_{\mathbb{S}^{d}} \frac{\xi_{*}^{t} M(\xi) \nabla_{\xi}(\widetilde{U} \widetilde{V})(\xi)}{\left(1-\xi_{d+1}\right)^{2}} \mathrm{~d} \xi
\end{aligned}
$$

where we use the identity $\xi \cdot \nabla_{\xi} \widetilde{V}(\xi)=0$. On the other hand, the following formula can easily be deduced

$$
\int_{\mathbb{S}^{d}} \widetilde{U}(\xi) e_{d+1} \cdot \nabla_{\xi} \widetilde{V}(\xi) \mathrm{d} \xi=-\int_{\mathbb{S}^{d}} \widetilde{V}(\xi) e_{d+1} \cdot \nabla_{\xi} \widetilde{U}(\xi) \mathrm{d} \xi+d \int_{\mathbb{S}^{d}} \xi_{d+1} \widetilde{U}(\xi) \widetilde{V}(\xi) \mathrm{d} \xi .
$$

Thus,

$$
\begin{aligned}
\int_{\mathbb{S}^{d}} \frac{\xi_{*}^{t} M(\xi) \nabla_{\xi}(\widetilde{U} \widetilde{V})(\xi)}{\left(1-\xi_{d+1}\right)^{2}} \mathrm{~d} \xi= & \int_{\mathbb{S}^{d}} \frac{e_{d+1} \cdot \nabla_{\xi}(\widetilde{U} \widetilde{V})(\xi)}{1-\xi_{d+1}} \mathrm{~d} \xi \\
= & -\int_{\mathbb{S}^{d}} \widetilde{U}(\xi) \widetilde{V}(\xi) \frac{e_{d+1} \cdot \nabla_{\xi} \xi_{d+1}}{\left(1-\xi_{d+1}\right)^{2}} \mathrm{~d} \xi \\
& +d \int_{\mathbb{S}^{d}} \widetilde{U}(\xi) \widetilde{V}(\xi) \frac{\xi_{d+1}}{1-\xi_{d+1}} \mathrm{~d} \xi \\
= & \int_{\mathbb{S}^{d}} \widetilde{U}(\xi) \widetilde{V}(\xi) \frac{(d-1) \xi_{d+1}-1}{\left(1-\xi_{d+1}\right)} \mathrm{d} \xi
\end{aligned}
$$

and

$$
\begin{aligned}
\mathscr{A}(u, v) & =\int_{\mathbb{S}^{d}} \nabla_{\xi} \widetilde{U}(\xi)^{t} \cdot \nabla_{\xi} \widetilde{V}(\xi)+\frac{d \alpha}{2} \int_{\mathbb{S}^{d}} \tilde{U}(\xi) \widetilde{V}(\xi) \mathrm{d} \xi \\
& =\int_{\mathbb{S}^{d}} \nabla_{\xi} \widehat{u}(\xi)^{t} \cdot \nabla_{\xi} \widehat{v}(\xi)+\frac{d \alpha}{2} \int_{\mathbb{S}^{d}} \widehat{u}(\xi) \widehat{v}(\xi) \mathrm{d} \xi .
\end{aligned}
$$

In particular, when $d \geq 2$, one has

$$
\begin{aligned}
\mathscr{A}\left(\mathscr{W}_{\ell, m}, \mathscr{W}_{r, s}\right) & =\int_{\mathbb{S}^{d}} \nabla_{\xi} \mathscr{Y}_{\ell, m}(\xi)^{t} \cdot \nabla_{\xi} \mathscr{Y}_{r, s}(\xi) \mathrm{d} \sigma(\xi)+\frac{\mathrm{d} \alpha}{2} \int_{\mathbb{S}^{d}} \mathscr{Y}_{\ell, m}(\xi) . \mathscr{Y}_{r, s}(\xi) \mathrm{d} \sigma(\xi) \\
& =\left(\ell(\ell+d-1)+\frac{d(d-2)}{4}\right) \int_{\mathbb{S}^{d}} \mathscr{Y}_{\ell, m}(\xi) \cdot \mathscr{Y}_{r, s}(\xi) \mathrm{d} \sigma(\xi) .
\end{aligned}
$$

This identity entails formula (3.25). 


\section{Appendix B. Formulae of Spherical harmonics on $\mathbb{S}^{2}$ AND ON $\mathbb{S}^{3}$}

A usual family of real spherical harmonics on $\mathbb{S}^{2}$ is given by

$$
\begin{aligned}
& Y_{\ell, 0}(\theta, \phi)=\sqrt{\frac{2 \ell+1}{2 \pi}} P_{\ell}^{0}(\cos \theta), \ell \geq 0, \\
& Y_{\ell, j}(\theta, \phi)=\sqrt{\frac{(2 \ell+1)(\ell-j) !}{2 \pi(\ell+j) !}} P_{\ell}^{j}(\cos \theta) \cos (j \phi), 1 \leq j \leq \ell, \\
& Z_{\ell, j}(\theta, \phi)=\sqrt{\frac{(2 \ell+1)(\ell-j) !}{2 \pi(\ell+j) !}} P_{\ell}^{j}(\cos \theta) \sin (j \phi), 1 \leq j \leq \ell .
\end{aligned}
$$

where $\left(P_{\ell}^{j}\right)$ are the associated Legendre functions defined as follows

$$
P_{\ell}^{j}(t)=\frac{(-1)^{j}}{2^{\ell} \ell !}\left(1-t^{2}\right)^{j / 2} \frac{\mathrm{d}^{\ell+j}}{\mathrm{~d} t^{\ell+j}}\left(1-t^{2}\right)^{\ell}, 0 \leq j \leq \ell .
$$

The family composed of functions $Y_{\ell, j}$ and $Z_{\ell, j}$ is orthonormal with respect to the scalar product of $L^{2}\left(\mathbb{S}^{2}\right)$. On $\mathbb{S}^{3}$, an orthonormal family of real spherical harmonics is given by

$$
\begin{aligned}
& U_{\ell k j}(x)=\frac{1}{\sqrt{a_{\ell, k}}}(\sin \chi)^{k} T_{\ell+1}^{(k+1)}(\cos \chi) Y_{k, j}(\theta, \phi), 0 \leq j \leq k \leq \ell \\
& S_{\ell k j}(x)=\frac{1}{\sqrt{a_{\ell, k}}}(\sin \chi)^{k} T_{\ell+1}^{(k+1)}(\cos \chi) Z_{k, t}(\theta, \phi), 1 \leq j \leq k \leq \ell
\end{aligned}
$$

where

$$
a_{\ell, k}=\frac{(\ell+1) \pi}{2} \frac{(k+\ell+1) !}{(\ell-k) !}
$$

\section{REFERENCES}

[1] C. Ahrens and G. Beylkin, Rotationally invariant quadratures for the sphere. Proc. R. Soc. London Ser. A Math. Phys. Eng. Sci. 465 (2009) 3103-3125.

[2] C. Amrouche, V. Girault and J. Giroire, Weighted Sobolev spaces for Laplace's equation in $\mathbf{R}^{n}$. J. Math. Pures Appl. $\mathbf{7 3}$ (1994) 579-606.

[3] N. Arar and T.Z. Boulmezaoud, Eigenfunctions of a weighted Laplace operator in the whole space. J. Math. Anal. Appl. 400 (2013) 161-173.

[4] K. Atkinson and W. Han, Spherical harmonics and approximations on the unit sphere: An introduction. Vol. 2044 of Lect. Notes Math. Springer, Heidelberg (2012).

[5] A. Bayliss and E. Turkel, Radiation boundary conditions for wavelike equations. Commun. Pure Appl. Math. 33 (1980) $707-725$.

[6] J.-P. Bérenger, A perfectly matched layer for absorption of electromagnetics waves. J. Comput. Phys. 114 (1994) 185-200.

[7] J.-P. Bérenger. Perfectly matched layer for the fdtd solution of wave-structure interaction problems. IEEE Trans. Antennas Propag. 44 (1996) 110-117,.

[8] Ch. Bernardi and Y. Maday, Approximations spectrales de problèmes aux limites elliptiques, Vol. 10 of Mathématiques \&\& Applications (Berlin) [Mathematics 83 Applications]. Springer-Verlag, Paris (1992).

[9] P. Bettess, Infinite elements. Int. J. Numer. Methods Engrg. 11 (1977) 53-64.

[10] P. Bettess and O. C. Zienkiewicz. Diffraction and refraction of surface waves using finite and infinite elements. Int. J. Numer. Methods Engrg. 11 (1977) 1271-1290.

[11] T.Z. Boulmezaoud. On the Laplace operator and on the vector potential problems in the half-space: an approach using weighted spaces. Math. Methods Appl. Sci. 26 (2003) 633-669.

[12] T.Z. Boulmezaoud, On the invariance of weighted Sobolev spaces under Fourier transform. C. R. Math. Acad. Sci. Paris 339 (2004) 861-866. 
[13] T.Z. Boulmezaoud, Inverted finite elements: a new method for solving elliptic problems in unbounded domains. ESAIM: M2AN 39 (2005) 109-145.

[14] T.Z. Boulmezaoud and K. Kaliche, A new numerical method for the model of solvation in continuum anisotropic dielectrics. In preparation (2015).

[15] T.Z. Boulmezaoud and U. Razafison, On the steady Oseen problem in the whole space. Hiroshima Math. J. 35 (2005) 371-401.

[16] T.Z. Boulmezaoud, S. Mziou and T. Boudjedaa, Numerical approximation of second-order elliptic problems in unbounded domains. J. Sci. Comput. 60 (2014) 295-312.

[17] J.P. Boyd, Orthogonal rational functions on a semi-infinite interval. J. Comput. Phys. 70 (1987) 63-88.

[18] J.P. Boyd, Spectral methods using rational basis functions on an infinite interval. J. Comput. Phys. 69 (1987) $112-142$.

[19] C.A. Brebbia, J.C.F. Telles and L.C. Wrobel, Boundary Element Techniques. Springer-Verlag, Berlin (1984).

[20] F. Brezzi, C. Johnson and J.-C. Nédélec, On the Coupling of Boundary Integral and Finite Element Methods. In Proc. of the Fourth Symposium on Basic Problems of Numerical Mathematics Plzevn. Charles Univ., Prague (1978) 103-114.

[21] D.S. Burnett, A three-dimensional acoustic infinite element based on a prolate spheroidal multipole expansion. J. Acoust. Soc. Amer. 96 (1994) 2798-2816.

[22] C. Canuto, S. I. Hariharan and L. Lustman, Spectral methods for exterior elliptic problems. Numer. Math. 46 (1985) 505-520.

[23] C. Carstensen, D. Zarrabi and E.P. Stephan, On the $h$-adaptive coupling of FE and BE for viscoplastic and elastoplastic interface problems. J. Comput. Appl. Math. 75 (1996) 345-363.

[24] J. Céa, Approximation variationnelle des problèmes aux limites. Ann. Inst. Fourier Grenoble 14 (1964) $345-444$.

[25] Ph.-G. Ciarlet, The finite element method for elliptic problems. North-Holland Publishing Co., Amsterdam (1978).

[26] D.L. Colton and R. Kressn, Integral equation methods in scattering theory. Pure Appl. Math. John Wiley \& Sons Inc., New York (1983).

[27] R. Cools, Constructing cubature formulae: the science behind the art. In vol. 6 of Acta Numer. Cambridge Univ. Press, Cambridge (1997) 1-54.

[28] M. Costabel and E.P. Stephan, Coupling of finite and boundary element methods for an elastoplastic interface problem. SIAM J. Numer. Anal. 27 (1990) 1212-1226.

[29] M. Costabel, V.J. Ervin and E.P. Stephan, Symmetric coupling of finite elements and boundary elements for a parabolic-elliptic interface problem. Quart. Appl. Math. 48 (1990) 265-279.

[30] B. Enquist and A. Majda, Absorbing boundary conditions for the numerical simulation of waves. Math. Comput. 31 (1977) 629-651.

[31] B. Enquist and A. Majda, Radiation boundary conditions for acoustic and elastic wave calculations. Commun. Pure Appl. Math. 32 (1979) 313-357.

[32] D. Funaro, Computational aspects of pseudospectral Laguerre approximations. Appl. Numer. Math. 6 (1990) $447-457$.

[33] D. Funaro and O. Kavian, Approximations of some diffusion evolution equations in unbounded domains by Hermite functions. Math. Comput. 57 (1990) 597-619.

[34] K. Gerdes and L. Demkowicz, Solution of 3D-Laplace and Helmholtz equations in exterior domains using $h p$-infinite elements. Comput. Methods Appl. Mech. Engrg. 137 (1996) 239-273.

[35] J. Giroire, Études de quelques problèmes aux limites extérieurs et résolution par équations intégrales. Ph.D. thesis, Université Pierre et Marie Curie, Paris (1987).

[36] J. Giroire and J.-C. Nédélec, Numerical solution of an exterior Neumann problem using a double layer potential. Math. Comput. 32 (1978) 973-990.

[37] B.-Y. Guo, J. Shen and Z.-Q. Wang, A rational approximation and its applications to differential equations on the half line. J. Sci. Comput. 15 (2000) 117-147.

[38] B. Hanouzet, Espaces de Sobolev avec poids application au problème de Dirichlet dans un demi espace. Rend. Sem. Mat. Univ. Padova 46 (1971) 227-272.

[39] W.J. Hehre, L. Radom, P.V.R. Schleyer and J.A. Pople, Ab initio molecular orbital theory. Wiley (1986).

[40] C. Johnson and J.-C. Nédélec, On the coupling of boundary integral and finite element methods. Math. Comput. 35 (1980) 1063-1079.

[41] Q.T. Le Gia and H.N. Mhaskar, Localized linear polynomial operators and quadrature formulas on the sphere. SIAM J. Numer. Anal. 47 (2008/09) 440-466.

[42] J. Lysmer and R.L. Kuhlemeyer, Finite difference model for infinite media. J. Eng. Mech. EMR 95 (1969) 859-877.

[43] Y. Maday, B. Pernaud-Thomas and H. Vandeven, Reappraisal of Laguerre type spectral methods. La Recherche Aerospatiale 6 (1985) 13-35.

[44] A.D. McLaren, Optimal numerical integration on a sphere. Math. Comput. 17 (1963) 361-383.

[45] C. Müller, Spherical harmonics. Vol. 17 of Lect. Notes Math. Springer-Verlag, Berlin (1966).

[46] C. Müller, Analysis of Spherical Symmetries in Euclidean Spaces. Vol. 129 of Applied Mathematical Sciences. Springer (1998).

[47] A. Ralston and Ph. Rabinowitz, A first course in numerical analysis, 2nd edition. Dover Publications, Inc., Mineola, New York (2001).

[48] R.T. Seeley, Spherical harmonics. Amer. Math. Monthly 73 (1966) 115-121,. 